\title{
Nitsche-XFEM with Streamline Diffusion Stabilization for a Two-Phase Mass Transport Problem
}

\author{
Christoph Lehrenfeld and Arnold Reusken*
}

\author{
Bericht Nr. $333 \quad$ November 2011
}

Key words: transport problem, Nitsche method, XFEM, streamline-diffusion stabilization

AMS subject classifications: $65 \mathrm{~N} 12,65 \mathrm{~N} 30$

Institut für Geometrie und Praktische Mathematik RWTH Aachen

Templergraben 55, D-52056 Aachen (Germany)

* Institut für Geometrie und Praktische Mathematik, RWTH-Aachen University, D-52056 Aachen, Germany; email: lehrenfeld@igpm.rwth-aachen.de, reusken@igpm.rwth-aachen.de 


\title{
NITSCHE-XFEM WITH STREAMLINE DIFFUSION STABILIZATION FOR A TWO-PHASE MASS TRANSPORT PROBLEM
}

\author{
CHRISTOPH LEHRENFELD AND ARNOLD REUSKEN*
}

\begin{abstract}
We consider an unsteady convection diffusion equation which models the transport of a dissolved species in two-phase incompressible flow problems. The so-called Henry interface condition leads to a jump condition for the concentration at the interface between the two phases. In [A. Hansbo, P. Hansbo, Comput. Methods Appl. Mech. Engrg. 191 (20002)], for the purely elliptic stationary case, extended finite elements (XFEM) are combined with a Nitsche-type of method, and optimal error bounds are derived. These results were extended to the unsteady case in [A. Reusken, T. Nguyen, J. Fourier Anal. Appl. 15 (2009)]. In the latter paper convection terms are also considered, but assumed to be small. In many two-phase flow applications, however, convection is the dominant transport mechanism. Hence there is a need for a stable numerical method for the case of a convection dominated transport equation. In this paper we address this topic and study the streamline diffusion stabilization for the Nitsche-XFEM method. The method is presented and results of numerical experiments are given that indicate that this kind of stabilization is satisfactory for this problem class. Furthermore, a theoretical error analysis of the stabilized Nitsche-XFEM method is presented that results in optimal a-priori discretization error bounds.
\end{abstract}

AMS subject classification. $65 \mathrm{~N} 12,65 \mathrm{~N} 30$

1. Introduction. Let $\Omega \subset \mathbb{R}^{d}, d=2,3$, be a convex polygonal domain that contains two different immiscible incompressible phases. The (in general time dependent) subdomains containing the two phases are denoted by $\Omega_{1}, \Omega_{2}$, with $\bar{\Omega}=\bar{\Omega}_{1} \cup \bar{\Omega}_{2}$. The interface $\Gamma:=\bar{\Omega}_{1} \cap \bar{\Omega}_{2}$ is assumed to be sufficiently smooth. A model example is a (rising) droplet in a flow field. The fluid dynamics in such a flow problem is usually modeled by the incompressible Navier-Stokes equations combined with suitable conditions at the interface which describe the effect of surface tension. For this model we refer to the literature, e.g. $[3,8,15,21,9]$. By w we denote the velocity field resulting from these Navier-Stokes equations. We assume that div $\mathbf{w}=0$ holds. Furthermore, we assume that the transport of the interface is determined by this velocity field, in the sense that $V_{\Gamma}=\mathbf{w} \cdot \mathbf{n}$ holds, where $V_{\Gamma}$ is the normal velocity of the interface and $\mathbf{n}$ denotes the unit normal at $\Gamma$ pointing from $\Omega_{1}$ into $\Omega_{2}$. In this paper we restrict ourselves to the case of a stationary interface, i.e., we assume $\mathbf{w} \cdot \mathbf{n}=0$. This case is (much) easier to handle than the case of an non-stationary interface $\Gamma=\Gamma(t)$. We restrict to this simpler case because even for that the issue of stabilization of the Nitsche-XFEM method for convection-dominated transport problems has not been investigated, yet. The case of a non-stationary interface will be studied in a forthcoming paper. We comment on this further in Remark 6 at the end of the paper. We consider a model which describes the transport of a dissolved species in a two-phase

\footnotetext{
*Institut für Geometrie und Praktische Mathematik, RWTH-Aachen University, D-52056 Aachen, Germany; email: lehrenfeld@igpm.rwth-aachen.de,reusken@igpm.rwth-aachen.de
} 
flow problem. In strong formulation this model is as follows:

$$
\begin{aligned}
\frac{\partial u}{\partial t}+\mathbf{w} \cdot \nabla u-\operatorname{div}(\varepsilon \nabla u) & =f \quad \text { in } \Omega_{i}, i=1,2, t \in[0, T], \\
{[\varepsilon \nabla u \cdot \mathbf{n}]_{\Gamma} } & =0, \\
{[\beta u]_{\Gamma} } & =0, \\
u(\cdot, 0) & =u_{0} \quad \text { in } \Omega_{i}, \quad i=1,2, \\
u(\cdot, t) & =0 \quad \text { on } \partial \Omega, \quad t \in[0, T] .
\end{aligned}
$$

For a sufficiently smooth function $v,[v]=[v]_{\Gamma}$ denotes the jump of $v$ across $\Gamma$, i.e. $[v]=\left(v_{1}\right)_{\mid \Gamma}-\left(v_{2}\right)_{\mid \Gamma}$, where $v_{i}=v_{\mid \Omega_{i}}$ is the restriction of $v$ to $\Omega_{i}$. In (1.1) we have standard parabolic convection-diffusion equations in the two subdomains $\Omega_{1}$ and $\Omega_{2}$. In most applications one has a homogeneous problem, i.e. $f \equiv 0$. The diffusion coefficient $\varepsilon=\varepsilon(x)$ is assumed to be piecewise constant:

$$
\varepsilon=\varepsilon_{i}>0 \text { in } \Omega_{i} .
$$

In general we have $\varepsilon_{1} \neq \varepsilon_{2}$. The interface condition in (1.2) results from the conservation of mass principle. The condition in (1.3) is the so-called Henry condition, cf. $[14,20,19,4,3]$. In this condition the coefficient $\beta=\beta(x)$ is strictly positive and piecewise constant:

$$
\beta=\beta_{i}>0 \text { in } \Omega_{i} .
$$

In general we have $\beta_{1} \neq \beta_{2}$, since species concentration usually has a jump discontinuity at the interface due to different solubilities within the respective fluid phases. Hence, the solution $u$ is discontinuous across the interface.

In recent years it has been shown that for such a transport problem with an (evolving) interface the Nitsche-XFEM method is very well suited [10, 17]. In [11, 12, $13,1,5]$ the application of the Nitsche-XFEM to other classes of problems is studied. In [10] this method is analyzed for a stationary heat diffusion problem (no convection) with a conductivity that is discontinuous across the interface $\left(\varepsilon_{1} \neq \varepsilon_{2}\right)$ but with a solution that is continuous across the interface $\left(\beta_{1}=\beta_{2}\right)$. In [17] the method is studied for the parabolic problem described above, with $\beta_{1} \neq \beta_{2}$ (discontinuous solution), and with a convection term in (1.1). It is assumed, however, that the transport problem is diffusion dominated. In none of these papers, or in other literature that we know of, the Nitsche-XFEM method is considered for a two-phase transport problem as in (1.1)-(1.5) that is convection-dominated. In this paper we treat this topic. We combine the Nitsche-XFEM method with one of the most popular FE stabilization techniques for convection-dominated problems, namely the streamline diffusion finite element method (SDFEM), cf. [18]. The resulting method is presented in section 2. In section 3 the method is applied to a convection-dominated test problem and its performance is investigated. An error analysis of the Nitsche-XFEM with SD stabilization is given in section 4 .

2. The Nitsche-XFEM method with SD stabilization. Since we restrict to the case of a stationary interface, the discontinuity in the solution is located at a fixed position, independent of $t$, which then allows a rather standard weak formulation and a corresponding discretization based on the method of lines approach. In this section we present this weak formulation and the stabilized Nitsche-XFEM discretization. In case of an evolving interface a space-time weak formulation and corresponding spacetime XFEM discretization is more natural, cf. Remark 6. 
We describe the Nitsche-XFEM method as treated in detail in [17]. We first introduce a suitable weak formulation of the transport problem. For this we need the space

$$
H_{0}^{1}\left(\Omega_{1} \cup \Omega_{2}\right):=\left\{v \in L^{2}(\Omega) \mid v_{\mid \Omega_{i}} \in H^{1}\left(\Omega_{i}\right), i=1,2, v_{\mid \partial \Omega}=0\right\} .
$$

For $v \in H_{0}^{1}\left(\Omega_{1} \cup \Omega_{2}\right)$ we write $v_{i}:=v_{\mid \Omega_{i}}, i=1,2$. Furthermore

$$
H:=L^{2}(\Omega), \quad V:=\left\{v \in H_{0}^{1}\left(\Omega_{1} \cup \Omega_{2}\right) \mid[\beta v]_{\Gamma}=0\right\} .
$$

Note:

$$
v \in V \Leftrightarrow \beta v \in H_{0}^{1}(\Omega) .
$$

On $H$ we use the scalar product

$$
(u, v)_{0}:=(\beta u, v)_{L^{2}}=\int_{\Omega} \beta u v d x
$$

which clearly is equivalent to the standard scalar product on $L^{2}(\Omega)$. The corresponding norm is denoted by $\|\cdot\|_{0}$. For $u, v \in H^{1}\left(\Omega_{i}\right)$ we define $(u, v)_{1, \Omega_{i}}:=$ $\beta_{i} \int_{\Omega_{i}} \nabla u_{i} \cdot \nabla v_{i} d x$ and furthermore

$$
(u, v)_{1, \Omega_{1} \cup \Omega_{2}}:=(u, v)_{1, \Omega_{1}}+(u, v)_{1, \Omega_{2}}, \quad u, v \in V .
$$

The corresponding norm is denoted by $|\cdot|_{1, \Omega_{1} \cup \Omega_{2}}$. This norm is equivalent to

$$
\left(\|\cdot\|_{0}^{2}+|\cdot|_{1, \Omega_{1} \cup \Omega_{2}}^{2}\right)^{\frac{1}{2}}=:\|\cdot\|_{1, \Omega_{1} \cup \Omega_{2}} .
$$

We emphasize that the norms $\|\cdot\|_{0}$ and $\|\cdot\|_{1, \Omega_{1} \cup \Omega_{2}}$ depend on $\beta$. We define the bilinear form

$$
a(u, v):=(\varepsilon u, v)_{1, \Omega_{1} \cup \Omega_{2}}+(\mathbf{w} \cdot \nabla u, v)_{0}, \quad u, v \in V .
$$

Consider the following weak formulation of the mass transport problem (1.1)-(1.5): Determine $u \in W^{1}(0, T ; V):=\left\{v \in L^{2}(0, T ; V) \mid v^{\prime} \in L^{2}\left(0, T ; V^{\prime}\right)\right\}$ such that $u(0)=$ $u_{0}$ and for almost all $t \in(0, T)$ :

$$
\left(\frac{d u}{d t}, v\right)_{0}+a(u, v)=(f, v)_{0} \quad \text { for all } v \in V .
$$

In [17] it is proved that if the velocity field $\mathbf{w}$ satisfies $\operatorname{div} \mathbf{w}=0$ in $\Omega_{i}, i=1,2$, $\mathbf{w} \cdot \mathbf{n}=0$ at $\Gamma$, and $\|\mathbf{w}\|_{L^{\infty}(\Omega)} \leq c<\infty$, then for $f \in H$, and $u_{0}$ sufficiently smooth the weak formulation (2.4) has a unique solution. For precise definitions of the generalized time derivatives used in the definition of $W^{1}(0, T ; V)$ and in $(2.4)$ we refer to [17].

We describe the Nitsche-XFEM method for spatial discretization of the weak formulation in (2.4). Let $\left\{\mathcal{T}_{h}\right\}_{h>0}$ be a family of shape regular triangulations of $\Omega$. A triangulation $\mathcal{T}_{h}$ consists of simplices $T$, with $h_{T}:=\operatorname{diam}(T)$ and $h:=\max \left\{h_{T} \mid T \in \mathcal{T}_{h}\right\}$. For any simplex $T \in \mathcal{T}_{h}$ let $T_{i}:=T \cap \Omega_{i}$ be the part of $T$ in $\Omega_{i}$. We now introduce the finite element space

$$
V_{h}^{\Gamma}:=\left\{v \in H_{0}^{1}\left(\Omega_{1} \cup \Omega_{2}\right) \mid v_{\mid T_{i}} \text { is linear for all } T \in \mathcal{T}_{h}, i=1,2 .\right\} .
$$


Note that $V_{h}^{\Gamma} \subset H_{0}^{1}\left(\Omega_{1} \cup \Omega_{2}\right)$, but $V_{h}^{\Gamma} \not \subset V$, since the Henry interface condition $\left[\beta v_{h}\right]=0$ does not necessarily hold for $v_{h} \in V_{h}^{\Gamma}$.

REMARK 1. In the literature a finite element discretization based on the space $V_{h}^{\Gamma}$ is often called an extended finite element method (XFEM), cf. [2, 6]. Furthermore, in the (engineering) literature this space is usually characterized in a different way, which we briefly explain. Let $V_{h} \subset H_{0}^{1}(\Omega)$ be the standard finite element space of continuous piecewise linears, corresponding to the triangulation $\mathcal{T}_{h}$. Define the index set $\mathcal{J}=\{1, \ldots, n\}$, where $n=\operatorname{dim} V_{h}$, and let $\left(\phi_{i}\right)_{i \in \mathcal{J}}$ be the nodal basis in $V_{h}$. Let $\mathcal{J}_{\Gamma}:=\left\{j \in \mathcal{J}|| \Gamma \cap \operatorname{supp}\left(\phi_{j}\right) \mid>0\right\}$ be the index set of those basis functions the support of which is intersected by $\Gamma$. The Heaviside function $H_{\Gamma}$ has the values $H_{\Gamma}(x)=0$ for $x \in \Omega_{1}, H_{\Gamma}(x)=1$ for $x \in \Omega_{2}$. Using this, for $j \in \mathcal{J}_{\Gamma}$ we introduce a so-called enrichment function $\Phi_{j}(x):=H_{\Gamma}(x)-H_{\Gamma}\left(x_{j}\right)$, where $x_{j}$ is the vertex with index $j$. We introduce new basis functions $\phi_{j}^{\Gamma}:=\phi_{j} \Phi_{j}, j \in \mathcal{J}_{\Gamma}$, and define the space

$$
V_{h} \oplus \operatorname{span}\left\{\phi_{j}^{\Gamma} \mid j \in \mathcal{J}_{\Gamma}\right\} .
$$

This space is the same as $V_{h}^{\Gamma}$ in (2.5) and the characterization in (2.6) accounts for the name "extended finite element method". The new basis functions $\phi_{j}^{\Gamma}$ have the property $\phi_{j}^{\Gamma}\left(x_{i}\right)=0$ for all $i \in \mathcal{J}$. An $L^{2}$-stability property of the basis $\left(\phi_{j}\right)_{j \in \mathcal{J}} \cup$ $\left(\phi_{j}^{\Gamma}\right)_{j \in J_{\Gamma}}$ of $V_{h}^{\Gamma}$ is given in [16].

Define

$$
\left(\kappa_{i}\right)_{\mid T}=\frac{\left|T_{i}\right|}{|T|}, \quad T \in \mathcal{T}_{h}, i=1,2,
$$

hence, $\kappa_{1}+\kappa_{2}=1$. For $v$ sufficiently smooth such that $\left(v_{i}\right)_{\mid \Gamma}, i=1,2$, are well-defined, we define the weighted average

$$
\{v\}:=\kappa_{1}\left(v_{1}\right)_{\mid \Gamma}+\kappa_{2}\left(v_{2}\right)_{\mid \Gamma} .
$$

For the average and jump operators the following identity holds for all $f, g$ such that these operators are well-defined:

$$
[f g]=\{f\}[g]+[f]\{g\}-\left(\kappa_{1}-\kappa_{2}\right)[f][g] .
$$

Define the scalar products

$$
(f, g)_{\Gamma}:=\int_{\Gamma} f g d s, \quad(f, g)_{\frac{1}{2}, h, \Gamma}:=\sum_{T \in \mathcal{T}_{h}^{\Gamma}} h_{T}^{-1} \int_{\Gamma_{T}} f g d s,
$$

where $\mathcal{T}_{h}^{\Gamma}$ is the collection of $T \in \mathcal{T}_{h}$ with $\Gamma_{T}=T \cap \Gamma \neq \emptyset$. With $\bar{\varepsilon}:=\frac{1}{2}\left(\varepsilon_{1}+\varepsilon_{2}\right)$ we introduce the bilinear form

$$
a_{h}(u, v):=a(u, v)-([\beta u],\{\varepsilon \nabla v \cdot \mathbf{n}\})_{\Gamma}-(\{\varepsilon \nabla u \cdot \mathbf{n}\},[\beta v])_{\Gamma}+\lambda \bar{\varepsilon}([\beta u],[\beta v])_{\frac{1}{2}, h, \Gamma},
$$

with $\lambda>0$ a parameter that will be specified below. Note that the scaling of the stabilization term $([\beta u],[\beta v])_{\frac{1}{2}, h, \Gamma}$ in $(2.8)$ differs from the standard one used in the literature for a diffusion dominated problem, which is of the form $\lambda([\beta u],[\beta v])_{\frac{1}{2}, h, \Gamma}$ with $\lambda$ a constant that is "sufficiently large". The theoretical analysis in section 4 motivates the following choice of $\lambda$ in (2.8):

$$
\lambda=\left\{\begin{array}{ccc}
c\|\mathbf{w}\|_{\infty} h / \bar{\varepsilon} & \text { if } \quad\|\mathbf{w}\|_{\infty} h \geq 2 \bar{\varepsilon} \\
c & \text { if } \quad\|\mathbf{w}\|_{\infty} h<2 \bar{\varepsilon}
\end{array}\right.
$$


with a constant $c$ that is sufficiently large, and $\|\mathbf{w}\|_{\infty}:=\|\mathbf{w}\|_{L^{\infty}(\Omega)}$. Note that in the convection-dominated case, i.e. $\|\mathbf{w}\|_{\infty} h \geq 2 \bar{\varepsilon}$, this results in a scaling of the stabilization term $([\beta u],[\beta v])_{\frac{1}{2}, h, \Gamma}$ that differs from the scaling with $c$ used in the diffusion dominated case.

REMARK 2. In practice the following localized variant of the parameter choice rule in (2.9) is used. For $T \in \mathcal{T}_{h}$ we define the element Peclet number $P_{h}^{T}:=$ $\frac{1}{2}\|\mathbf{w}\|_{\infty, T} h_{T} / \bar{\varepsilon}$. A generalization of the analysis in section 4 leads to the following choice of $\lambda=\lambda_{T}$ :

$$
\lambda_{T}=\left\{\begin{array}{ccc}
c\|\mathbf{w}\|_{\infty, T} h_{T} / \bar{\varepsilon} & \text { if } & P_{h}^{T} \geq 1 \\
c & \text { if } & P_{h}^{T}<1,
\end{array}\right.
$$

The stabilization term $\lambda \bar{\varepsilon}([\beta u],[\beta v])_{\frac{1}{2}, h, \Gamma}$ in $(2.8)$ is generalized to

$$
\bar{\varepsilon} \sum_{T \in \mathcal{T}_{h}^{\Gamma}} \lambda_{T} h_{T}^{-1} \int_{\Gamma_{T}}[\beta u][\beta v] d s .
$$

In practice this variant typically performs better than the one with a global stabilization parameter $\lambda$.

Using the bilinear form $a_{h}(\cdot, \cdot)$ we define a method of lines discretization of (2.4). Let $\hat{u}_{0} \in V_{h}^{\Gamma}$ be an approximation of $u_{0}$. For $t \in[0, T]$ let $u_{h}(t) \in V_{h}^{\Gamma}$ be such that $u_{h}(0)=\hat{u}_{0}$ and

$$
\left(\frac{d u_{h}}{d t}, v_{h}\right)_{0}+a_{h}\left(u_{h}, v_{h}\right)=\left(f, v_{h}\right)_{0} \text { for all } v_{h} \in V_{h}^{\Gamma} .
$$

Opposite to the weak formulation in (2.4), in this discretization method the Henry interface condition $\left[\beta u_{h}\right]=0$ is not treated as an "essential" interface condition in the finite element space $V_{h}^{\Gamma}$. This interface condition is satisfied only approximately by using a modified bilinear form $a_{h}(\cdot, \cdot)$, which is a technique due to Nitsche. For this semi-discretization optimal order error bounds are derived in [17]. In the analysis in that paper it is assumed that the transport problem is diffusion-dominated. In the evaluation of the bilinear form $a_{h}(\cdot, \cdot)$ one has to determine integrals over $\Gamma$. In practice the weak formulation will be used with $\Gamma$ replaced by an approximation $\Gamma_{h}$.

We now add the streamline diffusion stabilization to this semi-discretization. Recall that in a one-phase problem ( $\operatorname{set} \beta=1$ ) in the SD approach one adds a residual term of the form

$$
\sum_{T \in \mathcal{T}_{h}} \gamma_{T} \int_{T}\left(\frac{\partial u_{h}}{\partial t}+\mathbf{w} \cdot \nabla u_{h}-\operatorname{div}\left(\varepsilon \nabla u_{h}\right)-f\right)\left(\mathbf{w} \cdot \nabla v_{h}\right) d x
$$

to the variational formulation. The choice of the stabilization parameter value $\gamma_{T}$ is discussed below. If, as in our case, one considers linear finite elements then the term $\operatorname{div}\left(\varepsilon \nabla u_{h}\right)$ vanishes.

For the stabilization of the Nitsche-XFEM method we make obvious modifications related to the fact that in the XFEM space, close to the interface we have contributions on elements $T \cap \Omega_{i} \neq T$. For the stabilization we introduce a locally weighted discrete variant of $(\cdot, \cdot)_{0}$ :

$$
(u, v)_{0, h}:=\sum_{i=1}^{2} \sum_{T \in \mathcal{T}_{h}} \beta_{i} \gamma_{T} \int_{T \cap \Omega_{i}} u v d x=\sum_{T \in \mathcal{T}_{h}} \gamma_{T}(u, v)_{0, T}
$$


For the choice of $\gamma_{T}$ we use a strategy as in the standard finite element method, cf. $[18,7]$. We take $\gamma_{T}$ as follows:

$$
\gamma_{T}=\left\{\begin{array}{ccc}
\frac{2 h_{T}}{\|\mathbf{w}\|_{\infty}, T} & \text { if } & P_{h}^{T}>1 \\
h_{T}^{2} / \bar{\varepsilon} & \text { if } & P_{h}^{T} \leq 1
\end{array}\right.
$$

Very similar results (both in the theoretical analysis and in the experiments) are obtained if for the case $P_{h}^{T} \leq 1$ one sets $\gamma_{T}=0$. Note that the stabilization parameter $\gamma_{T}$ does not depend on the position of the interface within the element. We introduce the following Nitsche-XFEM semi-discretization method with SD stabilization: For $t \in[0, T]$ let $u_{h}(t) \in V_{h}^{\Gamma}$ be such that $u_{h}(0)=\hat{u}_{0}$ and

$$
\begin{aligned}
& \left(\frac{d u_{h}}{d t}, v_{h}\right)_{0}+\left(\frac{d u_{h}}{d t}, \mathbf{w} \cdot \nabla v_{h}\right)_{0, h}+a_{h}\left(u_{h}, v_{h}\right)+\left(\mathbf{w} \cdot \nabla u_{h}, \mathbf{w} \cdot \nabla v_{h}\right)_{0, h} \\
& =\left(f, v_{h}\right)_{0}+\left(f, \mathbf{w} \cdot \nabla v_{h}\right)_{0, h} \text { for all } v_{h} \in V_{h}^{\Gamma} .
\end{aligned}
$$

Clearly, this semi-discretization can be combined with standard methods for time discretization to obtain a fully discrete problem. For example, the $\theta$-scheme takes the following form, where for notational simplicity we assume that $f$ does not depend on $t$. For $n=0,1, \ldots, N-1$, with $N \Delta t=T$, set $u_{h}^{0}:=\hat{u}_{0}$ and determine $u_{h}^{n+1} \in V_{h}^{\Gamma}$ such that for all $v_{h} \in V_{h}^{\Gamma}$ :

$$
\begin{aligned}
& \left(\frac{u_{h}^{n+1}-u_{h}^{n}}{\Delta t}, v_{h}\right)_{0}+\left(\frac{u_{h}^{n+1}-u_{h}^{n}}{\Delta t}, \mathbf{w} \cdot \nabla v_{h}\right)_{0, h} \\
& +a_{h}\left(\theta u_{h}^{n+1}+(1-\theta) u_{h}^{n}, v_{h}\right)+\left(\mathbf{w} \cdot\left(\theta \nabla u_{h}^{n+1}+(1-\theta) \nabla u_{h}^{n}\right), \mathbf{w} \cdot \nabla v_{h}\right)_{0, h} \\
& =\left(f, v_{h}\right)_{0}+\left(f, \mathbf{w} \cdot \nabla v_{h}\right)_{0, h} .
\end{aligned}
$$

In the numerical experiments in section 3 we used this method with $\theta=1$.

REMARK 3. Above we considered the case of a stationary interface and an XFEM space based on piecewise linears. Both the Nitsche-XFEM method and the SD stabilization method presented above have a straightforward extension to higher order piecewise polynomials. Note that for higher order finite elements in the SD stabilization the term $\left(\operatorname{div}\left(\varepsilon \nabla u_{h}\right), \mathbf{w} \cdot \nabla v_{h}\right)_{0, h}$ has to be taken into account, cf. (2.12).

3. Numerical experiment. In this section we present results of a numerical experiment to illustrate properties of the stabilized Nitsche-XFEM method introduced above. The main goal is to compare the Nitsche-XFEM method with the stabilized Nitsche-XFEM method. Furthermore the effect of the choice of the stabilization parameter $\lambda$ in the Nitsche term is investigated.

3.1. Problem description. We consider a quasi two-dimensional time dependent problem with a stationary interface. The domain $\Omega:=[0,2] \times[0,2] \times[0,1] \subset \mathbb{R}^{3}$ is separated into a cylindrical domain $\Omega_{1}:=\left\{(x, y, z) \in \mathbb{R}^{3}:(x-1)^{2}+(y-1)^{2}<R^{2}\right\}$, with $R=0.25$, and $\Omega_{2}:=\Omega \backslash \Omega_{1}$ by the stationary interface $\Gamma:=\partial \Omega_{1} \backslash \partial \Omega$. The piecewise constant coefficients $\varepsilon, \beta$ are chosen as $\varepsilon=\left(\varepsilon_{1}, \varepsilon_{2}\right)=\left(10^{-4}, 2 \cdot 10^{-4}\right)$, $\beta=\left(\beta_{1}, \beta_{2}\right)=(3,1)$ and a stationary velocity field is given by

$$
\mathbf{w}=\left\{\begin{array}{clc}
\left(1+\frac{R^{2}\left(d_{y}^{2}-d_{x}^{2}\right)}{r^{4}}, \frac{-2 R^{2}\left(d_{x} d_{y}\right)}{r^{4}}, 0\right) & \text { if } & (x, y, z) \in \Omega_{2} \\
(0,0,0) & \text { if } \quad(x, y, z) \in \Omega_{1},
\end{array}\right.
$$

where $d_{x}:=x-1, d_{y}:=y-1$ and $r:=\left(d_{x}^{2}+d_{y}^{2}\right)^{\frac{1}{2}}$. A sketch of the domains and of $\mathbf{w}$ in term of field-lines is given in Fig. 3.1. 

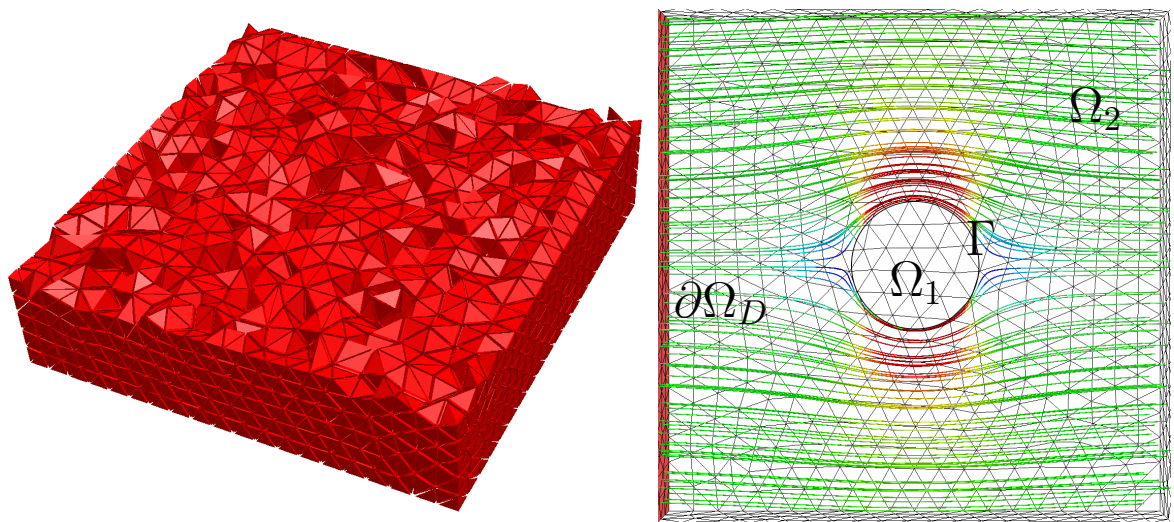

FIG. 3.1. Mesh and sketch of interface position and flow field

The assumptions on the velocity field made in section 1 are satisfied: $\operatorname{div} \mathbf{w}=0$ in both domains and $\mathbf{w} \cdot \mathbf{n}=0$ on $\Gamma$. We impose a Dirichlet boundary conditions on $\partial \Omega_{D}:=\{(x, y, z) \in \Omega: x=0\}$, s.t. $\left.u\right|_{\partial \Omega_{D}}=0.05$ and a homogeneous Neuman boundary condition $\varepsilon \nabla u \cdot \mathbf{n}=0$ on $\partial \Omega \backslash \partial \Omega_{D}$. As initial conditions we take $\left.u\right|_{t=0, \Omega_{1}}=$ $0,\left.u\right|_{t=0, \Omega_{2}}=0.05$. Note that these do not fulfill the interface condition (1.3).

This problem is strongly convection dominated since the ratio between the magnitude of the velocity field in $\Omega_{2}$ and the diffusion is of the order $10^{4}$. The physical Peclet number $\mathrm{P}_{D}:=\frac{\|\mathbf{w}\| 2 R}{\bar{\varepsilon}}$, which describes the relation between convective and diffuse mass transport, is around 3000 . Furthermore due to the inconsistent (w.r.t. condition (1.3)) initial conditions a boundary layer at the interface will form directly after $t=0$. With the help of standard perturbation theory (see e.g. [22]) one can show that this parabolic boundary layer has a thickness of size $O(\sqrt{\varepsilon t})$, independent of the velocity field. For $t \rightarrow \infty$ the solution converges to the stationary piecewise constant function $u=0.05 \beta^{-1}$. In Fig. 3.2 the solution along two lines is displayed, where one observes the predicted boundary layer behavior. In the experiment below we take $t=1$.

Summarizing, we consider a convection dominated, non-stationary transport problem with a stationary interface and with inconsistent initial values, resulting in parabolic boundary layers for small $t$.

3.2. Discretization. We use a shape regular quasi-uniform triangulation of $\Omega$ consisting of approx. 30000 tetrahedral elements such that the mesh is not aligned to the interface (cf. Fig. 3.1). With an average characteristic mesh size $h=0.1$ the mesh related Peclet number $\mathrm{P}_{h}^{T}$ reaches values up to 1000 . The mesh resolution is much too low to resolve the boundary layer (for $t=1$ ).

The exact interface $\Gamma$ is given by the zero level of the level set function $\varphi(x, y, z)=$ $(x-1)^{2}+(y-1)^{2}-0.25$. In the discretization we use a (sufficiently accurate) polygonal approximation $\Gamma_{h}$ of this interface. This introduces an additional error which is not analyzed here but is considered to be sufficiently small and to have negligible effect on the accuracy and stability properties of the (stabilized) Nitsche-XFEM method. We are primarily interested in the accuracy of the spatial discretization. Hence, in the implicit Euler method (2.16) we choose a small time step size $\Delta t=10^{-4}$, such that the total discretization error is dominated by the spatial discretization error.

We consider the following two variants of the Nitsche-XFEM method. Firstly, we use 


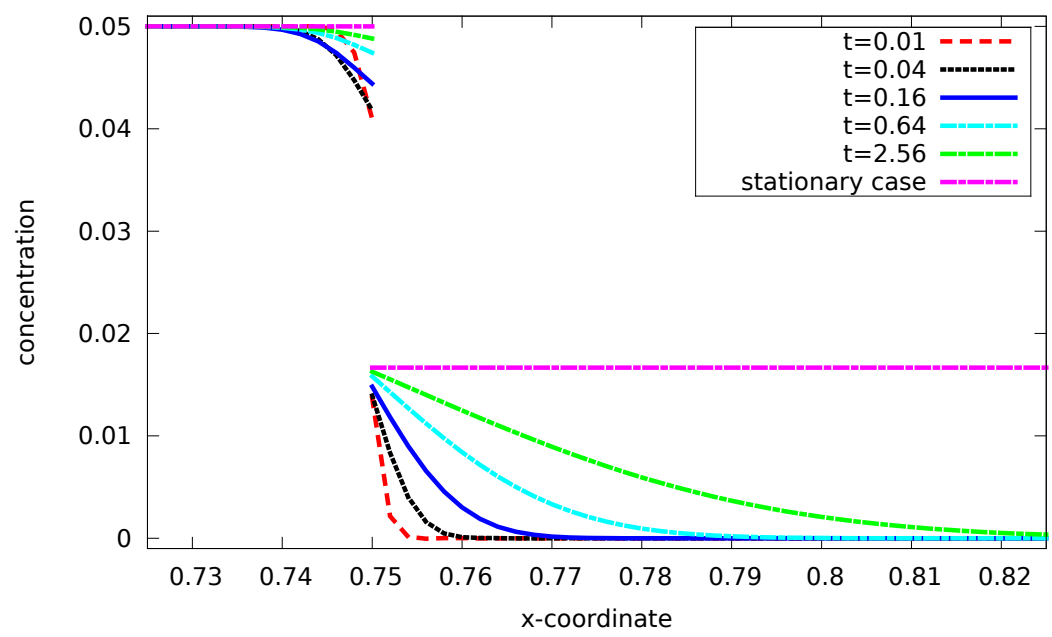

FIG. 3.2. Concentration profile through line $\gamma_{x}:=\{(x, y, z) \in \Omega: y=1, z=0.5\}$ for several values of $t$. One can observe the predicted $O(\sqrt{\varepsilon t})$ sized boundary layer.

the (standard) scaling $\lambda \bar{\varepsilon}$ of the Nitsche stabilization term, where we choose $\lambda=2$, which is sufficiently large in the sense of the analysis carried out in section 4 , i.e. it ensures coercivity of the bilinear form $a_{h}$ in (2.8). This scaling variant is denoted as the "diffusive" scaling as it is independent of the convection. In the second variant we take $\lambda=\lambda_{T}=c\|\mathbf{w}\|_{\infty, T} h_{T} / \bar{\varepsilon}$, according to (2.10) for $P_{h}^{T}>1$, with $c:=2$. Note that in this case the factor $\lambda \bar{\varepsilon}$ used as scaling for the Nitsche stabilization term does not depend on the diffusion coefficients $\varepsilon_{i}$. Hence we call this variant the "convective" scaling. In the experiments considered below the different scalings vary by approximately a factor 1000 .

For the streamline diffusion stabilized method, denoted by SD-Nitsche-XFEM, we consider the same two variants. We use a slightly different choice for the stabilization parameter $\gamma_{T}$ as in (2.14):

$$
\gamma_{T}=\left\{\begin{array}{ccc}
\left(1-\frac{1}{P_{h}^{T}}\right) \frac{h_{T}}{2\|\mathbf{w}\|_{\infty}, T} & \text { if } & P_{h}^{T}>1 \\
0 & \text { if } & P_{h}^{T} \leq 1 .
\end{array}\right.
$$

This choice can also be found in [7].

We computed a reference solution on a very fine $2 \mathrm{D}$ mesh which is aligned to the interface and resolves the boundary layer for $t>10^{-2}$. This reference solution is used to provide the profiles in Fig. 3.2 and the reference profiles in Fig. 3.4 below.

3.3. Numerical results. In Fig. 3.3 the numerical solution in the plane $z=0.5$ at $t=1$ (where the boundary layer has a width of approximately 0.01 ) is shown for four different methods. As a measure for the quality of the discretization of the interface condition $[\beta u]=0$ by the Nitsche method we use the $L^{2}$ norm of the jump $\left[\beta u_{h}\right]$ on the approximate interface $\Gamma_{h}$. In Fig. 3.3 we also give this error in the jump condition.

We observe several effects. Comparing the different scalings in the Nitsche method, i.e. the left and the right columns in Fig. 3.3, it is clear that the convective scaling performs much better. This scaling results in a stabilization parameter $\lambda \bar{\varepsilon}$ that is 


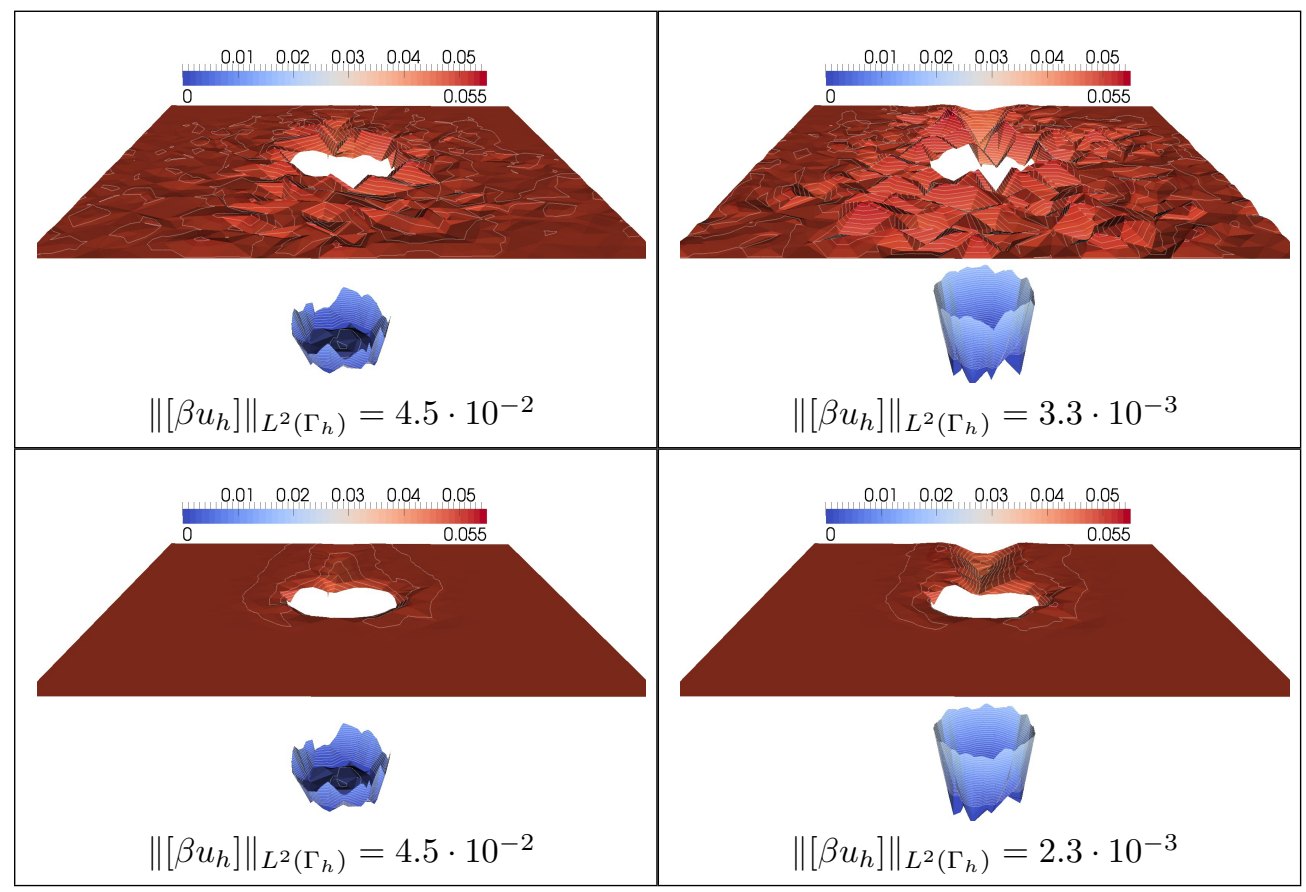

Fig. 3.3. Numerical solution in the plane $z=0.5$ at $t=1$ for Nitsche-XFEM (top) and SDNitsche-XFEM (bottom), with diffusion scaling (left) and convection scaling (right) of the Nitsche stabilization.

about a factor 1000 larger than with the diffusive scaling. Furthermore, from the results in the first row we conclude that the more weight is put on the interface condition, the larger the non-physical high oscillatory parts in the discrete solution are. This effect is as we expected, since for a more relaxed interface condition, the boundary layer and the corresponding instabilities in a standard Galerkin finite element method are triggered less. Finally, comparing the first and the second row, we see that the streamline diffusion stabilization suppresses the oscillations whereas the quality of the approximation of the interface condition is not negatively affected by this stabilization. We conclude that the SD-Nitsche-XFEM method with a convective scaling outperforms the three other methods and yields satisfactory results.

In Fig. 3.4 the numerical solutions of the same four methods as in Fig. 3.3 together with the reference solution, on the line $z=0.5, y=1.0$ in $\Omega_{2}$ at time $t=1$ are shown. One can observe that the boundary layer which is represented well by the reference solution is not resolved accurately by any of the four methods. Especially for $x>1.25$, i.e. downwind of $\Omega_{1}$ none of the methods come close to the reference solution. The solution $u_{h}$ of the SD-Nitsche-XFEM methods is much smoother than the other three and upwind of $\Omega_{1}$, where the solution is almost constant outside the boundary layer, it is very accurate. In Fig. 3.5 the results of the SD-Nitsche-XFEM method with convective scaling on three successive (uniformly) refined meshes are shown. The resolution of the boundary layer at $t=1$ improves if the grid is refined, but on level 3 the discrete solution is still not in good agreement with the reference solution. This is caused by the fact that large errors from smaller times, where the boundary layer is stronger and not well-resolved by the level 3 grid, are propagated in time. 


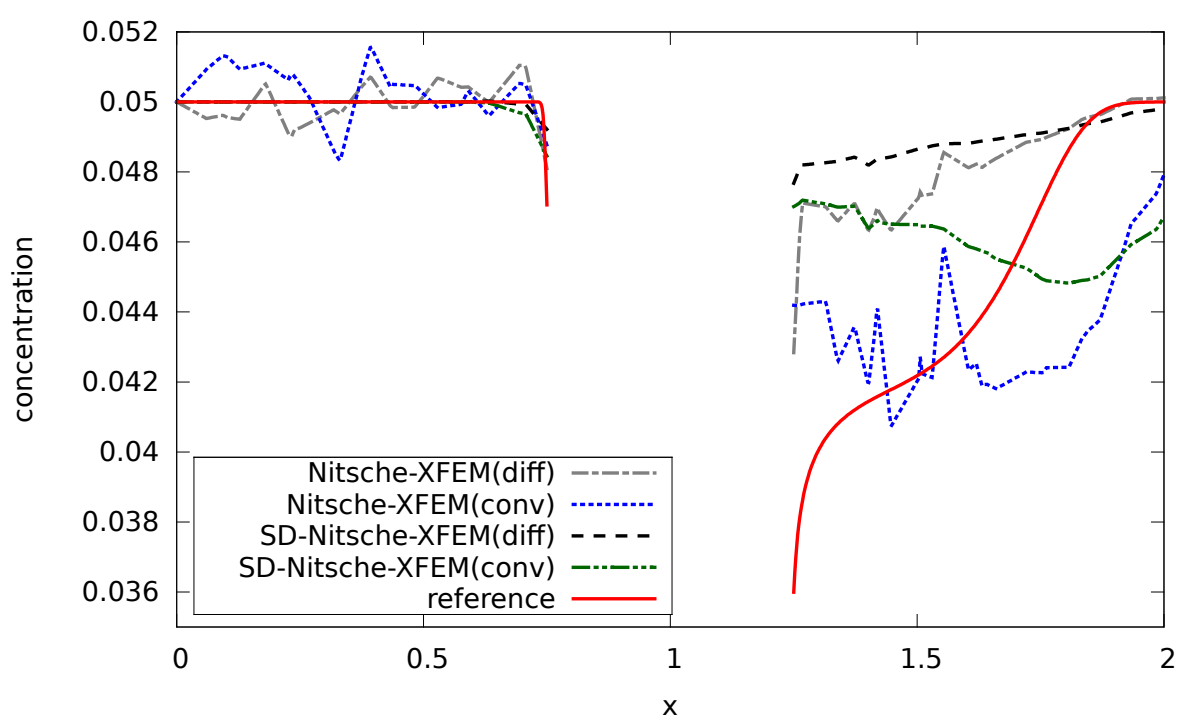

FIG. 3.4. Numerical solutions on the line $z=0.5, y=1.0$ at time $t=1$ obtained with NitscheXFEM, SD-Nitsche-XFEM, and the reference solution.

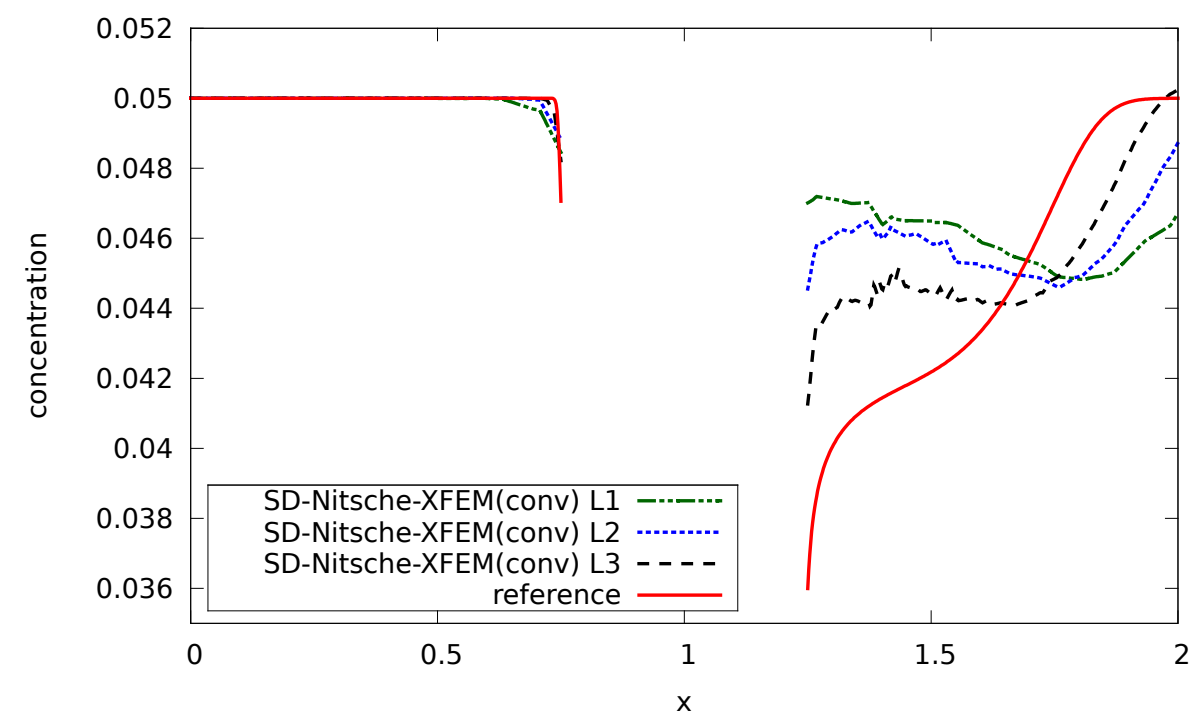

FIG. 3.5. Numerical solutions on the line $z=0.5, y=1.0$ at time $t=1$ obtained with SDNitsche-XFEM on three consecutively refined meshes and the reference solution.

REMARK 4. In the example considered above the instabilities were caused by non-matching (w.r.t. the Henry condition) initial condition and not by boundary conditions. It is well-known that the instabilities of the standard Galerkin method are very strong in cases with exponential boundary layers caused by Dirichlet boundary conditions. If we change the Neumann boundary condition at the outflow face $x=2$ to a homogeneous Dirichlet boundary condition an exponential boundary layer occurs 
at that face. This exponential layer of width $O(\varepsilon)$ is essentially independent of time. Therefore we consider the stationary case, i.e. $\frac{\partial u}{\partial t}=0$. We take diffusion coefficients $\varepsilon=\left(\varepsilon_{1}, \varepsilon_{2}\right)=\left(10^{-3}, 2 \cdot 10^{-3}\right)$ and apply the Nitsche-XFEM method with the diffusive scaling and the SD-Nitsche-XFEM method with the convective scaling. The numerical solutions obtained with these two methods are illustrated in the plane $z=0.5$ in Fig. 3.6. As expected, although the mesh Peclet number is only of order 100, for the method without stabilization the oscillations are much larger than in the case considered above, where the mesh Peclet number is of order 1000. With the SDNitsche-XFEM method the oscillations are almost completely eliminated and the error in the Henry condition is significantly smaller. Note that the approximation of the interface condition in this example is much better for the SD-Nitsche-XFEM method than in the previous example as the solution is almost piecewise constant at the interface.

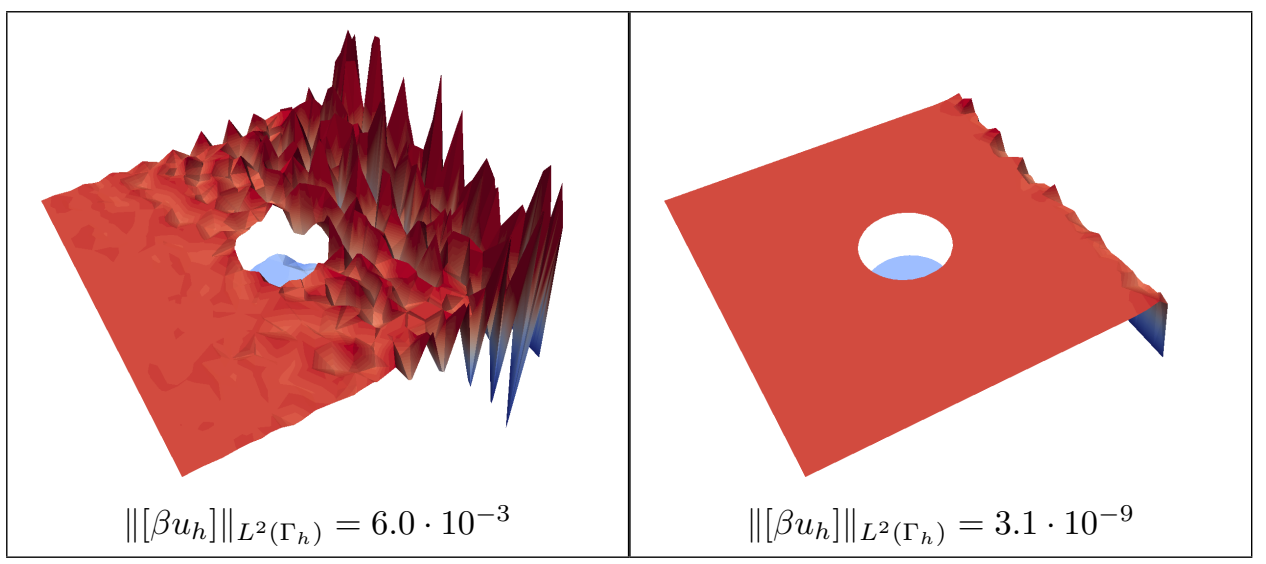

FIG. 3.6. Numerical solutions of the modified example (Remark 4) with Dirichlet boundary conditions at the outflow boundary obtained with NitscheXFEM (left) and SDNitscheXFEM (right)

4. Error analysis. In this section we present an error analysis of the NitscheXFEM with SD stabilization. We investigate the bilinear form

$$
\begin{aligned}
a_{h}(u, v) & :=(\varepsilon u, v)_{1, \Omega_{1} \cup \Omega_{2}}+(\mathbf{w} \cdot \nabla u, v)_{0}+\xi(u, v)_{0} \\
& -([\beta u],\{\varepsilon \nabla v \cdot \mathbf{n}\})_{\Gamma}-(\{\varepsilon \nabla u \cdot \mathbf{n}\},[\beta v])_{\Gamma}+\lambda \bar{\varepsilon}([\beta u],[\beta v])_{\frac{1}{2}, h, \Gamma} \\
& +(\xi u+\mathbf{w} \cdot \nabla u, \mathbf{w} \cdot \nabla u)_{0, h}
\end{aligned}
$$

on $W_{\text {reg }}+V_{h}^{\Gamma}$, with $V_{h}^{\Gamma}$ the XFEM space, cf. (2.5), and $W_{\text {reg }}:=\left\{u \in H_{0}^{1}\left(\Omega_{1} \cup\right.\right.$ $\left.\left.\Omega_{2}\right) \mid u_{\mid \Omega_{i}} \in H^{2}\left(\Omega_{i}\right), i=1,2\right\}$. Compared to the transport problem considered above we introduced an additional zero order term $\xi(u, v)_{0}$, with a given constant $\xi \geq 0$. This is standard in the analysis of convection-dominated problems (cf. [18]), since only if this zero order term is present $(\xi>0)$ one can derive uniform error bounds in the $L^{2}$-norm. We derive an error bound for the Galerkin projection of $u \in W_{\text {reg }}$ on the XFEM space $V_{h}^{\Gamma}$, cf. Theorem 4.7 below. We start with the main assumptions used and introduce additional notation. To obtain estimates that are uniform with respect to the parameter $\xi$, we have to generalize the choice of of the stabilization 
parameter $\gamma_{T}$. If $\xi=0$ we take $\gamma_{T}$ as in (2.14). For the case $\xi>0$ we take

$$
\gamma_{T}:=\left\{\begin{array}{cll}
\min \left\{\frac{1}{\xi}, \frac{2 h_{T}}{\|\mathbf{w}\|_{\infty}, T}\right\} & \text { if } & P_{h}^{T}>1 \\
\min \left\{\frac{1}{\xi}, \frac{h_{T}^{2}}{\bar{\varepsilon}}\right\} & \text { if } & P_{h}^{T} \leq 1 .
\end{array}\right.
$$

This parameter choice is essentially the same as in [18]. The following estimates can be derived:

$$
\gamma_{T} \xi \leq 1, \quad \gamma_{T}\|\mathbf{w}\|_{\infty, T} \leq 2 h_{T}, \quad \gamma_{T}^{-1} h_{T}^{2} \leq \xi h_{T}^{2}+\frac{1}{2}\|\mathbf{w}\|_{\infty, T} h_{T}+\bar{\varepsilon} .
$$

The family of triangulations $\left\{\mathcal{T}_{h}\right\}_{h>0}$ is assumed to be shape regular, but not necessarily quasi-uniform. The triangulation $\mathcal{T}_{h}$ is not assumed to be fitted to the interface $\Gamma$, but the resolution close to the interface should be sufficiently high such that the interface can be resolved by the triangulation, in the sense that if $\Gamma \cap T=: \Gamma_{T} \neq \emptyset$ then $\Gamma_{T}$ can be represented as the graph of a function on a planar cross-section of $T$ (cf. [10] for precise conditions). In the analysis of the Nitsche-XFEM method an interpolation operator $I_{h}^{\Gamma}: W_{\text {reg }} \rightarrow V_{h}^{\Gamma}$ plays an important role. We recall the definition of this operator. For $i=1,2$, let $R_{i}$ be the restriction operator to $\Omega_{i}$, i.e., $\left(R_{i} v\right)(x)=v(x)$ for $x \in \Omega_{i}$ and $\left(R_{i} v\right)(x)=0$ otherwise. Let $\mathcal{E}_{i}: H^{2}\left(\Omega_{i}\right) \rightarrow H^{2}(\Omega)$ be a bounded extension operator with $\mathcal{E}_{i} v=0$ on $\partial \Omega$, and $I_{h}: H^{2}(\Omega) \cap H_{0}^{1}(\Omega) \rightarrow V_{h}$ the standard nodal interpolation operator corresponding to the space $V_{h}$ of continuous linear finite elements. The XFEM interpolation operator is given by

$$
I_{h}^{\Gamma}=R_{1} I_{h} \mathcal{E}_{1} R_{1}+R_{2} I_{h} \mathcal{E}_{2} R_{2} .
$$

Define $T_{i}:=T \cap \Omega_{i}$. Note that $T_{i}$ can be very shape irregular. The constants that occur in the estimates in this section are independent of the shape regularity of $T_{i}$. For the interpolation operator $I_{h}^{\Gamma}$ optimal (local) interpolation error bounds can easily be derived (cf. $[10,16])$. The following holds:

$$
\begin{aligned}
\left\|u-I_{h}^{\Gamma} u\right\|_{H^{m}\left(T_{i}\right)} & \leq\left\|\mathcal{E}_{i} R_{i} u-I_{h} \mathcal{E}_{i} R_{i} u\right\|_{H^{m}(T)} \\
& \leq c h_{T}^{2-m}\left\|\mathcal{E}_{i} R_{i} u\right\|_{H^{2}(T)}, \quad m=0,1,2, \text { for } u \in W_{\text {reg }} .
\end{aligned}
$$

In the analysis below we use the assumptions $\operatorname{div} \mathbf{w}=0$ on $\Omega,\|\mathbf{w}\|_{L^{\infty}(\Omega)}<\infty$ and $\mathbf{w} \cdot \mathbf{n}=0$ on $\Gamma$.

We are particularly interested in the convection-dominated case, and therefore allow $\bar{\varepsilon}=\frac{1}{2}\left(\varepsilon_{1}+\varepsilon_{2}\right) \downarrow 0$, but we assume the ratio between $\varepsilon_{1}$ and $\varepsilon_{2}$ to be bounded, i.e. for $i=1,2$, we have $\bar{\varepsilon} / \varepsilon_{i} \leq c$ with a uniform (for $\bar{\varepsilon} \downarrow 0$ ) constant $c$.

We assume that the constants $\beta_{i}$ used in the Henry condition are of order one. We will need several norms related to the Nitsche stabilization and the streamline diffusion stabilization. The inner products $(\cdot, \cdot)_{0}$ and $(\cdot, \cdot)_{1, \Omega_{1} \cup \Omega_{2}}$ (with corresponding norms $\|\cdot\|_{0}$ and $\left.|\cdot|_{1, \Omega_{1} \cup \Omega_{2}}\right)$ have been defined above in section 2 . These inner products depend on a weighting with $\beta$, but this causes no problem since $\beta$ is assumed to be of order one. For the streamline diffusion stabilization we introduced the inner product $(u, v)_{0, h}=\sum_{T \in \mathcal{T}_{h}} \gamma_{T}(u, v)_{0, T}$ with corresponding norm denoted by $\|\cdot\|_{0, h}$. In the analysis of the Nitsche method the following norms are used:

$$
\|v\|_{\frac{1}{2}, h, \Gamma}^{2}=(v, v)_{\frac{1}{2}, h, \Gamma}=\sum_{T \in \mathcal{T}_{h}^{\Gamma}} h_{T}^{-1}\|v\|_{L^{2}\left(\Gamma_{T}\right)}^{2}, \quad\|v\|_{-\frac{1}{2}, h, \Gamma}^{2}:=\sum_{T \in \mathcal{T}_{h}^{\Gamma}} h_{T}\|v\|_{L^{2}\left(\Gamma_{T}\right)}^{2} .
$$

Recall that $\mathcal{T}_{h}^{\Gamma}$ is the collection of $T \in \mathcal{T}_{h}$ with $\Gamma_{T}=T \cap \Gamma \neq \emptyset$. We first derive interpolation error bounds in different norms, which turn out to be useful. 
The constants used in the results derived below are all independent of $\lambda, \xi, \bar{\varepsilon}, h,\|\mathbf{w}\|$, and of how the interface $\Gamma$ intersects the triangulation $\mathcal{T}_{h}$ (i.e. of the shape regularity of $\left.T_{i}\right)$.

Lemma 4.1. For $u \in W_{\text {reg }}$ the following interpolation error bounds hold:

$$
\begin{aligned}
\left\|u-I_{h}^{\Gamma} u\right\|_{0} & \leq c h^{2}\|u\|_{2, \Omega_{1} \cup \Omega_{2}} \\
\left|u-I_{h}^{\Gamma} u\right|_{1, \Omega_{1} \cup \Omega_{2}} & \leq c h\|u\|_{2, \Omega_{1} \cup \Omega_{2}} \\
\sqrt{\xi}\left\|u-I_{h}^{\Gamma} u\right\|_{0, h} & \leq c h^{2}\|u\|_{2, \Omega_{1} \cup \Omega_{2}} \\
\left\|\mathbf{w} \cdot \nabla\left(u-I_{h}^{\Gamma} u\right)\right\|_{0, h} & \leq c\|\mathbf{w}\|_{\infty}^{\frac{1}{2}} h^{1 \frac{1}{2}}\|u\|_{2, \Omega_{1} \cup \Omega_{2}} \\
\sum_{i=1}^{2}\left\|R_{i}\left(u-I_{h}^{\Gamma} u\right)\right\|_{\frac{1}{2}, h, \Gamma} & \leq c h\|u\|_{2, \Omega_{1} \cup \Omega_{2}} \\
\sum_{i=1}^{2}\left\|\mathbf{n} \cdot \nabla R_{i}\left(u-I_{h}^{\Gamma} u\right)\right\|_{-\frac{1}{2}, h, \Gamma} & \leq c h\|u\|_{2, \Omega_{1} \cup \Omega_{2} .}
\end{aligned}
$$

Proof. The results in (4.4), (4.5) are known in the literature, e.g. [10, 16]. For completeness we show how the result in (4.5) can be derived from (4.3):

$$
\begin{aligned}
\left|u-I_{h}^{\Gamma} u\right|_{1, \Omega_{1} \cup \Omega_{2}}^{2} & =\sum_{i=1}^{2} \sum_{T \in \mathcal{T}_{h}}\left|u-I_{h}^{\Gamma} u\right|_{1, T_{i}}^{2} \leq c \sum_{i=1}^{2} \sum_{T \in \mathcal{T}_{h}} h_{T}^{2}\left\|\mathcal{E}_{i} R_{i} u\right\|_{2, T}^{2} \\
& \leq c h^{2} \sum_{i=1}^{2}\left\|\mathcal{E}_{i} R_{i} u\right\|_{2, \Omega}^{2} \leq c h^{2} \sum_{i=1}^{2}\left\|R_{i} u\right\|_{2, \Omega_{i}}^{2}=c h^{2}\|u\|_{2, \Omega_{1} \cup \Omega_{2}}^{2} .
\end{aligned}
$$

The result in (4.4) can be proved using similar arguments. Using the choice of the stabilization parameter $\gamma_{T}$ we obtain

$$
\xi\left\|u-I_{h}^{\Gamma} u\right\|_{0, h}^{2}=\sum_{T \in \mathcal{T}_{h}} \xi \gamma_{T}\left\|u-I_{h}^{\Gamma} u\right\|_{0, T}^{2} \leq\left\|u-I_{h}^{\Gamma} u\right\|_{0}^{2} \leq c h^{4}\|u\|_{2, \Omega_{1} \cup \Omega_{2}}^{2},
$$

and thus the result in (4.6) holds. The result in (4.7) follows from

$$
\begin{aligned}
\left\|\mathbf{w} \cdot \nabla\left(u-I_{h}^{\Gamma} u\right)\right\|_{0, h}^{2} & =\sum_{T \in \mathcal{T}_{h}} \gamma_{T}\left\|\mathbf{w} \cdot \nabla\left(u-I_{h}^{\Gamma} u\right)\right\|_{0, T}^{2} \leq \sum_{i=1}^{2} \sum_{T \in \mathcal{T}_{h}} \gamma_{T}\|\mathbf{w}\|_{\infty, T}^{2}\left|u-I_{h}^{\Gamma} u\right|_{1, T_{i}}^{2} \\
& \leq c\|\mathbf{w}\|_{L^{\infty}(\Omega)} h\left|u-I_{h}^{\Gamma} u\right|_{1, \Omega_{1} \cup \Omega_{2}}^{2} \leq c\|\mathbf{w}\|_{L^{\infty}(\Omega)} h^{3}\|u\|_{2, \Omega_{1} \cup \Omega_{2}}^{2} .
\end{aligned}
$$

The results in (4.8), (4.9), are derived in [10]. The essential ingredient is the following result:

$$
\|w\|_{L^{2}\left(\Gamma_{T}\right)}^{2} \leq c\left(h_{T}^{-1}\|w\|_{L^{2}(T)}^{2}+h_{T}|w|_{1, T}^{2}\right) \quad \text { for all } w \in H^{1}(T)
$$

which holds for all $T \in \mathcal{T}_{h}^{\Gamma}$ and with a constant $c$ that is independent of the shape 
regularity of $T_{i}$, cf. [10,9]. For completeness we give a proof of (4.8):

$$
\begin{aligned}
& \sum_{i=1}^{2}\left\|R_{i}\left(u-I_{h}^{\Gamma} u\right)\right\|_{\frac{1}{2}, h, \Gamma}^{2}=\sum_{i=1}^{2} \sum_{T \in \mathcal{T}_{h}^{\Gamma}} h_{T}^{-1}\left\|\mathcal{E}_{i} R_{i} u-I_{h} \mathcal{E}_{i} R_{i} u\right\|_{L^{2}\left(\Gamma_{T}\right)}^{2} \\
& \leq c \sum_{i=1}^{2} \sum_{T \in \mathcal{T}_{h}^{\Gamma}}\left(h_{T}^{-2}\left\|\mathcal{E}_{i} R_{i} u-I_{h} \mathcal{E}_{i} R_{i} u\right\|_{L^{2}(T)}^{2}+\left|\mathcal{E}_{i} R_{i} u-I_{h} \mathcal{E}_{i} R_{i} u\right|_{1, T}^{2}\right) \\
& \leq c h^{2} \sum_{i=1}^{2} \sum_{T \in \mathcal{T}_{h}^{\Gamma}}\left\|\mathcal{E}_{i} R_{i} u\right\|_{2, T}^{2} \leq c h^{2} \sum_{i=1}^{2}\left\|\mathcal{E}_{i} R_{i} u\right\|_{2, \Omega}^{2} \\
& \leq c h^{2} \sum_{i=1}^{2}\left\|R_{i} u\right\|_{2, \Omega_{i}}^{2}=c h^{2}\|u\|_{2, \Omega_{1} \cup \Omega_{2}}^{2} .
\end{aligned}
$$

The result in (4.9) can be proved with similar arguments.

As we will see below, we can derive an ellipticity and continuity result for the bilinear form $a_{h}(\cdot, \cdot)$ with respect to a suitable norm. As expected this norm involves terms that come from the Nitsche stabilization and from the streamline diffusion stabilization. To simplify the presentation we split the bilinear form in two parts (corresponding to Nitsche and streamline diffusion stabilization) and first consider these two parts separately. Afterwards the results for these two parts can easily be glued together. We use the splitting

$$
\begin{aligned}
a_{h}(u, v)= & a_{h}^{N}(u, v)+a_{h}^{S D}(u, v) \\
a_{h}^{N}(u, v)= & \frac{1}{2}(\varepsilon u, v)_{1, \Omega_{1} \cup \Omega_{2}}-([\beta u],\{\varepsilon \nabla v \cdot \mathbf{n}\})_{\Gamma}-(\{\varepsilon \nabla u \cdot \mathbf{n}\},[\beta v])_{\Gamma} \\
& +\lambda \bar{\varepsilon}([\beta u],[\beta v])_{\frac{1}{2}, h, \Gamma} \\
a_{h}^{S D}(u, v)= & \frac{1}{2}(\varepsilon u, v)_{1, \Omega_{1} \cup \Omega_{2}}+(\mathbf{w} \cdot \nabla u, v)_{0}+\xi(u, v)_{0}+(\xi u+\mathbf{w} \cdot \nabla u, \mathbf{w} \cdot \nabla u)_{0, h} .
\end{aligned}
$$

Corresponding norms are defined as

$$
\begin{aligned}
\|v\|_{N}^{2} & =\frac{1}{2} \bar{\varepsilon}|v|_{1, \Omega_{1} \cup \Omega_{2}}^{2}+\lambda \bar{\varepsilon}\|[\beta v]\|_{\frac{1}{2}, h, \Gamma}^{2} \\
\|v\|_{S D}^{2} & =\frac{1}{2} \bar{\varepsilon}|v|_{1, \Omega_{1} \cup \Omega_{2}}^{2}+\xi\|v\|_{0}^{2}+\|\mathbf{w} \cdot \nabla v\|_{0, h}^{2} .
\end{aligned}
$$

Lemma 4.2. There exists a constant $c>0$ such that

$$
a_{h}^{S D}\left(v_{h}, v_{h}\right) \geq c\left\|v_{h}\right\|_{S D}^{2} \quad \text { for all } v_{h} \in V_{h}^{\Gamma} .
$$

Proof. We apply partial integration to the term $\left(\mathbf{w} \cdot \nabla v_{h}, v_{h}\right)_{0}$. Since $v_{h}$ may be discontinuous across $\Gamma$ we have to split the integral. Using $\mathbf{w} \cdot \mathbf{n}=0, \operatorname{div} \mathbf{w}=0$ and $v_{h}(x)=0$ for $x \in \partial \Omega$ we obtain

$$
\begin{aligned}
\left(\mathbf{w} \cdot \nabla v_{h}, v_{h}\right)_{0} & =\sum_{i=1}^{2} \int_{\Omega_{i}} \beta_{i} \mathbf{w} \cdot \nabla v_{h} v_{h} d x=\sum_{i=1}^{2} \int_{\partial \Omega_{i} \cap \partial \Omega} \beta_{i} v_{h}^{2} \mathbf{w} \cdot \mathbf{n}_{\Omega} d s \\
& +\int_{\Gamma}\left[\beta v_{h}^{2}\right] \mathbf{w} \cdot \mathbf{n} d s-\sum_{i=1}^{2} \int_{\Omega_{i}} \beta_{i} \mathbf{w} \cdot \nabla v_{h} v_{h}+\beta_{i}(\operatorname{div} \mathbf{w}) v_{h}^{2} d x \\
& =-\left(\mathbf{w} \cdot \nabla v_{h}, v_{h}\right)_{0} .
\end{aligned}
$$


Hence, $\left(\mathbf{w} \cdot \nabla v_{h}, v_{h}\right)_{0}=0$ holds. Furthermore, using $\gamma_{T} \xi \leq 1$ we get

$$
\begin{aligned}
\xi\left(v_{h}, \mathbf{w} \cdot \nabla v_{h}\right)_{0, h} & =\xi \sum_{T \in \mathcal{T}_{h}} \gamma_{T}\left(v_{h}, \mathbf{w} \cdot \nabla v_{h}\right)_{0, T} \\
& \leq \frac{1}{2} \sum_{T \in \mathcal{T}_{h}} \xi^{2} \gamma_{T}\left\|v_{h}\right\|_{0, T}^{2}+\gamma_{T}\left\|\mathbf{w} \cdot \nabla v_{h}\right\|_{0, T}^{2} \\
& \leq \frac{1}{2} \xi\left\|v_{h}\right\|_{0}^{2}+\frac{1}{2}\left\|\mathbf{w} \cdot \nabla v_{h}\right\|_{0, h}^{2} .
\end{aligned}
$$

Hence,

$$
\begin{aligned}
a_{h}^{S D}\left(v_{h}, v_{h}\right) & \geq \frac{1}{2} \min \left\{\varepsilon_{1}, \varepsilon_{2}\right\}\left\|v_{h}\right\|_{1, \Omega_{1} \cup \Omega_{2}}^{2}+\xi\left\|v_{h}\right\|_{0}^{2}+\left\|\mathbf{w} \cdot \nabla v_{h}\right\|_{0, h}^{2}+\xi\left(v_{h}, \mathbf{w} \cdot \nabla v_{h}\right)_{0, h} \\
& \geq c \bar{\varepsilon}\left\|v_{h}\right\|_{1, \Omega_{1} \cup \Omega_{2}}^{2}+\frac{1}{2} \xi\left\|v_{h}\right\|_{0}^{2}+\frac{1}{2}\left\|\mathbf{w} \cdot \nabla v_{h}\right\|_{0, h}^{2}
\end{aligned}
$$

with a constant $c>0$ which depends only on the ratio between $\varepsilon_{1}$ and $\varepsilon_{2}$, which is assumed to be bounded.

LEMma 4.3. There exists a constant $c$ such that

$a_{h}^{S D}\left(u-I_{h}^{\Gamma} u, v_{h}\right) \leq c\left(\sqrt{\bar{\varepsilon}}+\sqrt{\|\mathbf{w}\|_{\infty} h}+\sqrt{\xi} h\right) h\|u\|_{2, \Omega_{1} \cup \Omega_{2}}\left\|v_{h}\right\|_{S D} \quad \forall u \in W_{\text {reg }}, v_{h} \in V_{h}^{\Gamma}$.

Proof. We use the notation $e_{h}:=u-I_{h}^{\Gamma} u$ and recall the definition of $a_{h}^{S D}(\cdot, \cdot)$ :

$a_{h}^{S D}\left(e_{h}, v_{h}\right)=\frac{1}{2}\left(\varepsilon e_{h}, v_{h}\right)_{1, \Omega_{1} \cup \Omega_{2}}+\left(\mathbf{w} \cdot \nabla e_{h}, v_{h}\right)_{0}+\xi\left(e_{h}, v_{h}\right)_{0}+\left(\xi e_{h}+\mathbf{w} \cdot \nabla e_{h}, \mathbf{w} \cdot \nabla v_{h}\right)_{0, h}$.

Using the interpolation error bounds of lemma 4.1 we obtain

$$
\begin{aligned}
\frac{1}{2}\left(\varepsilon e_{h}, v_{h}\right)_{1, \Omega_{1} \cup \Omega_{2}} & \leq c \sqrt{\bar{\varepsilon}} h\|u\|_{2, \Omega_{1} \cup \Omega_{2}}\left\|v_{h}\right\|_{S D} \\
\xi\left(e_{h}, v_{h}\right)_{0} & \leq c \sqrt{\xi} h^{2}\|u\|_{2, \Omega_{1} \cup \Omega_{2}}\left\|v_{h}\right\|_{S D} \\
\xi\left(e_{h}, \mathbf{w} \cdot \nabla v_{h}\right)_{0, h} & \leq c \sqrt{\xi} h^{2}\|u\|_{2, \Omega_{1} \cup \Omega_{2}}\left\|v_{h}\right\|_{S D} \\
\left(\mathbf{w} \cdot \nabla e_{h}, \mathbf{w} \cdot \nabla v_{h}\right)_{0, h} & \leq c\|\mathbf{w}\|_{\infty}^{\frac{1}{2}} h^{1 \frac{1}{2}}\|u\|_{2, \Omega_{1} \cup \Omega_{2}}\left\|v_{h}\right\|_{S D} .
\end{aligned}
$$

To the term $\left(\mathbf{w} \cdot \nabla e_{h}, v_{h}\right)_{0}$ we apply partial integration as in (4.10), resulting in

$$
\begin{aligned}
\left(\mathbf{w} \cdot \nabla e_{h}, v_{h}\right)_{0} & =-\left(e_{h}, \mathbf{w} \cdot \nabla v_{h}\right)_{0} \leq\left(\sum_{T \in \mathcal{T}_{h}} \gamma_{T}^{-1}\left\|e_{h}\right\|_{0, T}^{2}\right)^{\frac{1}{2}}\left\|v_{h}\right\|_{S D} \\
& \leq c\left(\xi h^{2}+\|\mathbf{w}\|_{\infty} h+\bar{\varepsilon}\right)^{\frac{1}{2}} h\|u\|_{2, \Omega_{1} \cup \Omega_{2}}\left\|v_{h}\right\|_{S D},
\end{aligned}
$$

where in the last inequality we used the bound for $\gamma_{T}^{-1} h_{T}^{2}$ given in (4.2). Combining these estimates completes the proof.

We now turn to the analysis of the Nitsche bilinear form $a_{h}^{N}(\cdot, \cdot)$. We need the following inverse inequality given in [10]. For completeness we include its elementary proof.

LemmA 4.4. There exists a constant $c_{I}$ independent of $\bar{\varepsilon}$ such that

$$
\left\|\left\{\varepsilon \nabla v_{h} \cdot \mathbf{n}\right\}\right\|_{-\frac{1}{2}, h, \Gamma} \leq c_{I} \bar{\varepsilon}\left|v_{h}\right|_{1, \Omega_{1} \cup \Omega_{2}} \quad \text { for all } v_{h} \in V_{h}^{\Gamma} .
$$


Proof. Let $P_{k}$ be the space of polynomials of degree at most $k$. Using a scaling argument it follows that there exists a constant $c$, depending only on $k$ and the shape regularity of the triangulation $\mathcal{T}_{h}$, such that

$$
h_{T} \kappa_{i}\|p\|_{L^{2}\left(\Gamma_{T}\right)}^{2} \leq c\|p\|_{L^{2}\left(T_{i}\right)}^{2} \quad \text { for al } p \in P_{k}, T \in \mathcal{T}_{h}^{\Gamma}, i=1,2 .
$$

Using this we get

$$
\begin{aligned}
& \left\|\left\{\varepsilon \nabla v_{h} \cdot \mathbf{n}\right\}\right\|_{-\frac{1}{2}, h, \Gamma}^{2} \leq 2 \sum_{i=1}^{2} \kappa_{i}^{2}\left\|\varepsilon_{i} \nabla\left(v_{h}\right)_{i} \cdot \mathbf{n}\right\|_{-\frac{1}{2}, h, \Gamma}^{2} \\
& =2 \sum_{i=1}^{2} \sum_{T \in \mathcal{T}_{h}^{\Gamma}} h_{T} \kappa_{i}^{2}\left\|\varepsilon_{i} \nabla\left(v_{h}\right)_{i} \cdot \mathbf{n}\right\|_{L^{2}\left(\Gamma_{T}\right)}^{2} \leq c \bar{\varepsilon} \sum_{i=1}^{2} \sum_{T \in \mathcal{T}_{h}^{\Gamma}}\left|v_{h}\right|_{1, T_{i}}^{2} \leq c \bar{\varepsilon}\left|v_{h}\right|_{1, \Omega_{1} \cup \Omega_{2}}^{2},
\end{aligned}
$$

and thus the result holds. $\square$

We now derive an ellipticity result for $a_{h}^{N}(\cdot, \cdot)$ :

LemmA 4.5. There exist constants $c_{1}>0, c_{s}>0$ such that for $\lambda>c_{s}$

$$
a_{h}^{N}\left(v_{h}, v_{h}\right) \geq c_{1}\left\|v_{h}\right\|_{N}^{2} \quad \text { for all } v_{h} \in V_{h}^{\Gamma} .
$$

Proof. Define $\hat{c}=\frac{1}{2 \varepsilon} \min \left\{\varepsilon_{1}, \varepsilon_{2}\right\} \leq \frac{1}{2}$ and take $\lambda \geq 4 c_{I}^{2} \hat{c}^{-1}$ with $c_{I}$ from lemma 4.4. The following holds:

$$
\begin{aligned}
a_{h}^{N}\left(v_{h}, v_{h}\right) & \geq \hat{c} \bar{\varepsilon}\left|v_{h}\right|_{1, \Omega_{1} \cup \Omega_{2}}^{2}-2\left\|\left[\beta v_{h}\right]\right\|_{\frac{1}{2}, h, \Gamma}\left\|\left\{\varepsilon \nabla v_{h} \cdot \mathbf{n}\right\}\right\|_{-\frac{1}{2}, h, \Gamma}+\lambda \bar{\varepsilon}\left\|\left[\beta v_{h}\right]\right\|_{\frac{1}{2}, h, \Gamma}^{2} \\
& \geq \hat{c} \bar{\varepsilon}\left|v_{h}\right|_{1, \Omega_{1} \cup \Omega_{2}}^{2}-2 c_{I} \bar{\varepsilon}\left\|\left[\beta v_{h}\right]\right\|_{\frac{1}{2}, h, \Gamma}\left|v_{h}\right|_{1, \Omega_{1} \cup \Omega_{2}}+\lambda \bar{\varepsilon}\left\|\left[\beta v_{h}\right]\right\|_{\frac{1}{2}, h, \Gamma}^{2} \\
& \geq \frac{1}{2} \hat{c} \bar{\varepsilon}\left|v_{h}\right|_{1, \Omega_{1} \cup \Omega_{2}}^{2}+\left(\lambda-2 c_{I}^{2} \hat{c}^{-1}\right) \bar{\varepsilon}\left\|\left[\beta v_{h}\right]\right\|_{\frac{1}{2}, h, \Gamma}^{2} \\
& \geq \frac{1}{2} \hat{c} \bar{\varepsilon}\left|v_{h}\right|_{1, \Omega_{1} \cup \Omega_{2}}^{2}+\frac{1}{2} \lambda \bar{\varepsilon}\left\|\left[\beta v_{h}\right]\right\|_{\frac{1}{2}, h, \Gamma}^{2} \geq \hat{c}\left\|v_{h}\right\|_{N}^{2}
\end{aligned}
$$

$\square$

Lemma 4.6. There exists a constant $c$ such that for $\lambda>0$

$$
a_{h}^{N}\left(u-I_{h}^{\Gamma} u, v_{h}\right) \leq c \sqrt{\bar{\varepsilon}}\left(\sqrt{\lambda}+\frac{1}{\sqrt{\lambda}}\right) h\|u\|_{2, \Omega_{1} \cup \Omega_{2}}\left\|v_{h}\right\|_{N}
$$

holds for all $u \in W_{\text {reg }}, v_{h} \in V_{h}^{\Gamma}$.

Proof. We use the notation $e_{h}:=u-I_{h}^{\Gamma} u$ and recall the definition of $a_{h}^{N}(\cdot, \cdot)$ :

$$
\begin{aligned}
a_{h}^{N}\left(e_{h}, v_{h}\right)= & \frac{1}{2}\left(\varepsilon e_{h}, v_{h}\right)_{1, \Omega_{1} \cup \Omega_{2}}-\left(\left[\beta e_{h}\right],\left\{\varepsilon \nabla v_{h} \cdot \mathbf{n}\right\}\right)_{\Gamma}-\left(\left\{\varepsilon \nabla e_{h} \cdot \mathbf{n}\right\},\left[\beta v_{h}\right]\right)_{\Gamma} \\
& +\lambda \bar{\varepsilon}\left(\left[\beta e_{h}\right],\left[\beta v_{h}\right]\right)_{\frac{1}{2}, h, \Gamma} .
\end{aligned}
$$

Using the interpolation error bounds of lemma 4.1 and the inverse inequality in 
lemma 4.4 we obtain

$$
\begin{aligned}
\frac{1}{2}\left(\varepsilon e_{h}, v_{h}\right)_{1, \Omega_{1} \cup \Omega_{2}} & \leq c \sqrt{\bar{\varepsilon}} h\|u\|_{2, \Omega_{1} \cup \Omega_{2}}\left\|v_{h}\right\|_{N} \\
-\left(\left[\beta e_{h}\right],\left\{\varepsilon \nabla v_{h} \cdot \mathbf{n}\right\}\right)_{\Gamma} & \leq\left\|\left[\beta e_{h}\right]\right\|_{\frac{1}{2}, h, \Gamma}\left\|\left\{\varepsilon \nabla v_{h} \cdot \mathbf{n}\right\}\right\|_{-\frac{1}{2}, h, \Gamma} \\
& \leq c \bar{\varepsilon} \sum_{i=1}^{2}\left\|R_{i} e_{h}\right\|_{\frac{1}{2}, h, \Gamma}\left|v_{h}\right|_{1, \Omega_{1} \cup \Omega_{2}} \leq c \sqrt{\bar{\varepsilon}} h\|u\|_{2, \Omega_{1} \cup \Omega_{2}}\left\|v_{h}\right\|_{N} \\
-\left(\left\{\varepsilon \nabla e_{h} \cdot \mathbf{n}\right\},\left[\beta v_{h}\right]\right)_{\Gamma} & \leq\left\|\left\{\varepsilon \nabla e_{h} \cdot \mathbf{n}\right\}\right\|_{-\frac{1}{2}, h, \Gamma}\left\|\left[\beta v_{h}\right]\right\|_{\frac{1}{2}, h, \Gamma} \\
& \leq c \bar{\varepsilon} \sum_{i=1}^{2}\left\|\mathbf{n} \cdot \nabla R_{i} e_{h}\right\|_{-\frac{1}{2}, h, \Gamma}\left\|\left[\beta v_{h}\right]\right\|_{\frac{1}{2}, h, \Gamma} \\
& \leq c \sqrt{\frac{\bar{\varepsilon}}{\lambda}} h\|u\|_{2, \Omega_{1} \cup \Omega_{2}}\left\|v_{h}\right\|_{N} \\
\lambda \bar{\varepsilon}\left(\left[\beta e_{h}\right],\left[\beta v_{h}\right]\right)_{\frac{1}{2}, h, \Gamma} & \leq \lambda \bar{\varepsilon}\left\|\left[\beta e_{h}\right]\right\|_{\frac{1}{2}, h, \Gamma}\left\|\left[\beta v_{h}\right]\right\|_{\frac{1}{2}, h, \Gamma} \leq c \sqrt{\bar{\varepsilon} \lambda} h\|u\|_{2, \Omega_{1} \cup \Omega_{2}}\left\|v_{h}\right\|_{N} .
\end{aligned}
$$

Combination of these estimates and using $\frac{1}{\sqrt{\lambda}}+\sqrt{\lambda}>1$ proves the result. $\square$

We now combine the estimates derived above for $a_{h}^{N}(\cdot, \cdot)$ and $a_{h}^{S D}(\cdot, \cdot)$. For this we introduce the norm

$$
\|v\|^{2}=\|v\|_{N}^{2}+\|v\|_{S D}^{2}=\bar{\varepsilon}|v|_{1, \Omega_{1} \cup \Omega_{2}}^{2}+\xi\|v\|_{0}^{2}+\|\mathbf{w} \cdot \nabla v\|_{0, h}^{2}+\lambda \bar{\varepsilon}\|[\beta v]\|_{\frac{1}{2}, h, \Gamma}^{2} .
$$

Note that the two terms $\|\mathbf{w} \cdot \nabla v\|_{0, h}^{2}$ and $\lambda \bar{\varepsilon}\|[\beta v]\|_{\frac{1}{2}, h, \Gamma}^{2}$ originate from the stabilization terms in the streamline diffusion and the Nitsche method, respectively.

We discuss the choice of the stabilization parameter $\lambda$ in the Nitsche method. For the error analysis in the norm $\|\cdot\|$ it is natural to balance the upper bounds in lemma 4.3 and in lemma 4.6. For simplicity we make the (weak) assumption that $\xi h^{2} \leq c\left(\bar{\varepsilon}+\|\mathbf{w}\|_{\infty} h\right)$ holds, i.e. in the bound in lemma 4.3 the factor $\sqrt{\bar{\varepsilon}}+\sqrt{\|\mathbf{w}\|_{\infty} h}+$ $\sqrt{\xi} h$ can be replaced by $\sqrt{\bar{\varepsilon}}+\sqrt{\|\mathbf{w}\|_{\infty} h}$. The factor $\sqrt{\bar{\varepsilon}}(\sqrt{\lambda}+1 / \sqrt{\lambda})$ in the upper bound in lemma 4.6 should balance the latter factor, i.e.,

$$
\sqrt{\bar{\varepsilon}}+\sqrt{\|\mathbf{w}\|_{\infty} h} \approx \sqrt{\bar{\varepsilon}}(\sqrt{\lambda}+1 / \sqrt{\lambda})
$$

This leads to the choice as in (2.9), namely $\lambda=c\|\mathbf{w}\|_{\infty} h / \bar{\varepsilon}$ if $\|\mathbf{w}\|_{\infty} h \geq \bar{\varepsilon}$ and $\lambda=c$ otherwise. We take $c=c_{s}$, cf. lemma 4.5, to guarantee ellipticity. A slightly refined error analysis leads to a localized variant of this parameter choice given in remark 2 . In the remainder we take $\lambda$ as in (2.9), with $c=c_{s}$, cf. lemma 4.5. To simplify the presentation, in the estimates below the terms $\|\mathbf{w}\|_{\infty}$ are absorbed in the constant $c$.

From the interpolation error bounds in lemma 4.1, and using $\lambda \bar{\varepsilon} \leq c\left(\bar{\varepsilon}+\|\mathbf{w}\|_{\infty} h\right) \leq$ $c(\bar{\varepsilon}+h)$, we obtain

$$
\left\|u-I_{h}^{\Gamma} u\right\| \leq c(\sqrt{\bar{\varepsilon}}+\sqrt{h}+\sqrt{\xi} h) h\|u\|_{2, \Omega_{1} \cup \Omega_{2}} \quad \text { for all } u \in W_{\mathrm{reg}} .
$$

THEOREM 4.7. For $u \in W_{\text {reg }}$ let $R_{G} u \in V_{h}^{\Gamma}$ be the Galerkin projection for the bilinear form $a_{h}(\cdot, \cdot)$, i.e. $a_{h}\left(R_{G} u, v_{h}\right)=a_{h}\left(u, v_{h}\right)$ for all $v_{h} \in V_{h}^{\Gamma}$. The following holds:

$$
\left\|u-R_{G} u\right\| \leq c(\sqrt{\bar{\varepsilon}}+\sqrt{h}+\sqrt{\xi} h) h\|u\|_{2, \Omega_{1} \cup \Omega_{2}} \quad \text { for all } u \in W_{\text {reg }} .
$$


The constant $c$ is independent of $\bar{\varepsilon}, h, \xi$ and of how the interface $\Gamma$ intersects the triangulation $\mathcal{T}_{h}$.

Proof. The proof uses standard arguments. Define $\chi_{h}=R_{G} u-I_{h}^{\Gamma} u \in V_{h}^{\Gamma}$. Using the results in the lemmas above we obtain, with a suitable $c>0$,

$$
\begin{aligned}
\left\|\chi_{h}\right\|^{2} & =\left\|\chi_{h}\right\|_{N}^{2}+\left\|\chi_{h}\right\|_{S D}^{2} \leq c\left(a_{h}^{N}\left(\chi_{h}, \chi_{h}\right)+a_{h}^{S D}\left(\chi_{h}, \chi_{h}\right)\right) \\
& =c a_{h}\left(\chi_{h}, \chi_{h}\right)=c a_{h}\left(u-I_{h}^{\Gamma} u, \chi_{h}\right)=c a_{h}^{N}\left(u-I_{h}^{\Gamma} u, \chi_{h}\right)+c a_{h}^{S D}\left(u-I_{h}^{\Gamma} u, \chi_{h}\right) \\
& \leq c(\sqrt{\bar{\varepsilon}}+\sqrt{h}+\sqrt{\xi} h) h\|u\|_{2, \Omega_{1} \cup \Omega_{2}}\left\|\chi_{h}\right\| .
\end{aligned}
$$

The result follows from a triangle inequality and the interpolation error bound in (4.12).

We comment on the bound derived in (4.13). For the diffusion dominated case, i.e. $\bar{\varepsilon} \sim 1$, this result reduces to results known in the literature. We discuss the convection dominated case $\bar{\varepsilon} \leq h$ with $\xi \in[0,1]$ and write $e_{h}:=u-R_{G} u$. Furthermore we assume $h \leq c h_{T}$ (quasi-uniformity of the family of triangulations). Using $h \leq c \gamma_{T}$ for all $T \in \mathcal{T}_{h}$ we obtain from (4.13)

$$
\left\|\mathbf{w} \cdot \nabla e_{h}\right\|_{L^{2}(\Omega)} \leq \operatorname{ch}\|u\|_{2, \Omega_{1} \cup \Omega_{2}} .
$$

Hence, as for the streamline diffusion finite element method with the standard linear finite element space, we have an optimal error bound (uniformly in $\bar{\varepsilon}$ ) for the derivative of the error in streamline direction. Furthermore, from

$$
\lambda \bar{\varepsilon}\left\|\left[\beta e_{h}\right]\right\|_{\frac{1}{2}, h, \Gamma}^{2} \leq c h^{3}\|u\|_{2, \Omega_{1} \cup \Omega_{2}}^{2},
$$

and $\lambda \bar{\varepsilon}=c h$, we obtain

$$
\left\|\left[\beta e_{h}\right]\right\|_{L^{2}(\Gamma)} \leq \operatorname{ch}^{1 \frac{1}{2}}\|u\|_{2, \Omega_{1} \cup \Omega_{2}}
$$

uniformly in $\bar{\varepsilon}$. This bound of order $h^{1 \frac{1}{2}}$ for the error in the jump approximation is the same as for the diffusion dominated case. Finally, if we take $\xi>0$ we obtain an $L^{2}$-norm error bound that is the same as for the streamline diffusion finite element method with the standard linear finite element space, namely

$$
\left\|e_{h}\right\|_{L^{2}(\Omega)} \leq \frac{c}{\sqrt{\xi}} h^{1 \frac{1}{2}}\|u\|_{2, \Omega_{1} \cup \Omega_{2}} .
$$

REMARK 5. As noted in Remark 3, the SD-Nitsche-XFEM method has a straightforward extension to finite elements of higher order. We comment on the generalization of the error analysis presented above to the higher order case. The interpolation error bounds in Lemma 4.1 can easily be generalized to higher order extended finite elements. The result in Lemma 4.4, cf. (4.11), also holds for higher order elements, and using this the results for the Nitsche bilinear form in the Lemmas 4.5 and 4.6 can be generalized. In the analysis of the streamline diffusion bilinear form, however, a difficulty arises related to an inverse inequality needed in the analysis. For higher order finite elements the term $\left(\operatorname{div}\left(\varepsilon \nabla u_{h}\right), \mathbf{w} \cdot \nabla v_{h}\right)_{0, h}$ arises in the streamline diffusion stabilization. In the analysis of the streamline diffusion method for a standard higher order finite element space $V_{h}$ one uses an inverse inequality of the form $\left\|\Delta v_{h}\right\|_{0, T} \leq \mu_{\text {inv }} h_{T}^{-1}\left|v_{h}\right|_{1, T}$ for all $v_{h} \in V_{h}$, cf. [18]. Such a result does not hold in a higher order XFEM space, since the supports $T_{i}=T \cap \Omega_{i}$ of the additional (discontinuous) basis functions can be very shape irregular. We only have 
$\left\|\Delta v_{h}\right\|_{0, T_{i}} \leq \mu\left(T_{i}\right) h_{T_{i}}^{-1}\left|v_{h}\right|_{1, T_{i}}$ with a factor $\mu\left(T_{i}\right)$ that depends on the shape regularity of $T_{i}$. To control this, instead of (4.1), one can choose a stabilization parameter $\gamma_{T_{i}}$ that is sufficiently small. This would yield a stability result as in Lemma 4.2. If, however, this parameter is "too small" it is not likely that a result as in Lemma 4.3, which uses the third inequality in (4.2), still holds. We did not investigate this further.

5. Note on mass conservation. We derive a mass conservation property of the stabilized semi-discretization in (2.15) for the case of a stationary interface, i.e. $\mathbf{w} \cdot \mathbf{n}=0$. For this we replace the homogeneous Dirichlet boundary condition in (1.5) by a, in view of mass conservation, more natural one. Define the inflow boundary by $\partial \Omega_{-}:=\left\{x \in \partial \Omega \mid \mathbf{w} \cdot \mathbf{n}_{\Omega}<0\right\}$, where $\mathbf{n}_{\Omega}$ is the outward pointing unit normal on $\partial \Omega$, and $\partial \Omega_{+}:=\partial \Omega \backslash \partial \Omega_{-}$. Instead of (1.5) we consider

$$
\begin{array}{r}
(u \mathbf{w}-\varepsilon \nabla u) \cdot \mathbf{n}_{\Omega}=u_{I} \mathbf{w} \cdot \mathbf{n}_{\Omega} \quad \text { on } \partial \Omega_{-}, t \in[0, T] \\
\varepsilon \nabla u \cdot \mathbf{n}_{\Omega}=0 \quad \text { on } \partial \Omega_{+}, t \in[0, T],
\end{array}
$$

with $u_{I}$ a given mass inflow function. In the weak formulation we have to change accordingly the function space $V$ to $\tilde{V}:=\left\{v \in H^{1}\left(\Omega_{1} \cup \Omega_{2}\right) \mid[\beta v]_{\Gamma}=0\right\}$, and the weak formulation is as follows, cf. (2.4): Determine $u \in W^{1}(0, T ; \tilde{V})$ such that $u(0)=u_{0}$ and for almost all $t \in(0, T)$ :

$$
\left(\frac{d u}{d t}, v\right)_{0}+a(u, v)-\int_{\partial \Omega_{-}} \beta u v \mathbf{w} \cdot \mathbf{n}_{\Omega} d s=(f, v)_{0}-\int_{\partial \Omega_{-}} \beta u_{I} v \mathbf{w} \cdot \mathbf{n}_{\Omega} d s \text { for all } v \in \tilde{V} .(5.2)
$$

Using $\mathbf{w} \cdot \mathbf{n}=0$ on $\Gamma$ and $\operatorname{div} \mathbf{w}=0$ in $\Omega_{i}$ we get

$$
\begin{aligned}
\left(\mathbf{w} \cdot \nabla u, \beta^{-1}\right)_{0} & =\int_{\Omega} \mathbf{w} \cdot \nabla u d x=\sum_{i=1}^{2}\left(\int_{\partial \Omega_{i}} u \mathbf{w} \cdot \mathbf{n}_{\Omega_{i}} d s-\int_{\Omega_{i}} u \operatorname{div} \mathbf{w} d x\right) \\
& =\int_{\partial \Omega_{-}} u \mathbf{w} \cdot \mathbf{n}_{\Omega} d s+\int_{\partial \Omega_{+}} u \mathbf{w} \cdot \mathbf{n}_{\Omega} d s .
\end{aligned}
$$

Using this and taking the test function $v=\frac{1}{\beta} \in \tilde{V}$ in (5.2) we obtain the global mass conservation property

$$
\frac{d}{d t} \int_{\Omega} u d x+\int_{\partial \Omega_{-}} u_{I} \mathbf{w} \cdot \mathbf{n}_{\Omega} d s+\int_{\partial \Omega_{+}} u \mathbf{w} \cdot \mathbf{n}_{\Omega} d s=\int_{\Omega} f d x .
$$

This relation can also be derived directly from the strong formulation in (1.1), by integrating the equations in (1.1) over $\Omega$, and using the relations in (1.2), (5.1), (5.3). We show that an analogon of the conservation law (5.4) holds for the NitscheXFEM stabilized discretization. Due to the modification of the boundary condition the XFEM space we use is given by $\tilde{V}_{h}^{\Gamma}:=\left\{v \in H^{1}\left(\Omega_{1} \cup \Omega_{2}\right) \mid v_{\mid T_{i}}\right.$ is linear for all $T \in$ $\left.\mathcal{T}_{h}, i=1,2.\right\}$ and the discretization in $(2.15)$ is modified as follows: determine $u_{h}(t) \in$ $\tilde{V}_{h}^{\Gamma}$ such that $u_{h}(0)=\hat{u}_{0}$ and

$$
\begin{aligned}
& \left(\frac{d u_{h}}{d t}, v_{h}\right)_{0}+\left(\frac{d u_{h}}{d t}, \mathbf{w} \cdot \nabla v_{h}\right)_{0, h}+a_{h}\left(u_{h}, v_{h}\right)+\left(\mathbf{w} \cdot \nabla u_{h}, \mathbf{w} \cdot \nabla v_{h}\right)_{0, h} \\
& -\int_{\partial \Omega_{-}} \beta u_{h} v_{h} \mathbf{w} \cdot \mathbf{n}_{\Omega} d s=\left(f, v_{h}\right)_{0}+\left(f, \mathbf{w} \cdot \nabla v_{h}\right)_{0, h}-\int_{\partial \Omega_{-}} \beta u_{I} v_{h} \mathbf{w} \cdot \mathbf{n}_{\Omega} d s
\end{aligned}
$$

for all $v_{h} \in \tilde{V}_{h}^{\Gamma}$. For the discrete conservation property it is essential that the XFEM space contains piecewise smooth functions that are allowed to be discontinuous across 
$\Gamma$. In particular the piecewise constant function $\beta^{-1}$ is contained in $\tilde{V}_{h}^{\Gamma}$. Taking this test function $v_{h}$ in (5.5) all terms with $\nabla v_{h}$ vanish and for the Nitsche bilinear form, cf. (2.8), we have $a_{h}\left(u_{h}, \beta^{-1}\right)=\left(\mathbf{w} \cdot \nabla u_{h}, \beta^{-1}\right)_{0}$. Thus we obtain

$$
\frac{d}{d t} \int_{\Omega} u_{h} d x+\int_{\Omega} \mathbf{w} \cdot \nabla u_{h} d x-\int_{\partial \Omega_{-}} u_{h} \mathbf{w} \cdot \mathbf{n}_{\Omega} d s=\int_{\Omega} f d x-\int_{\partial \Omega_{-}} u_{I} \mathbf{w} \cdot \mathbf{n}_{\Omega} d s .
$$

Using partial integration for the term $\int_{\Omega} \mathbf{w} \cdot \nabla u_{h} d x$, as in (5.3), results in

$$
\frac{d}{d t} \int_{\Omega} u_{h} d x+\int_{\partial \Omega_{-}} u_{I} \mathbf{w} \cdot \mathbf{n}_{\Omega} d s+\int_{\partial \Omega_{+}} u_{h} \mathbf{w} \cdot \mathbf{n}_{\Omega} d s=\int_{\Omega} f d x
$$

which is the discrete global mass conservation analogon of the one in (5.4). Finally we briefly consider a mass conservation property in each subdomain $\Omega_{i}$. We assume $\partial \Omega_{1} \cap \partial \Omega=\emptyset$, hence, $\partial \Omega_{1}=\Gamma$. From (1.1) we get

$$
\frac{d}{d t} \int_{\Omega_{1}} u d x-\int_{\Gamma} \varepsilon \nabla u \cdot \mathbf{n} d s=\int_{\Omega_{1}} f d x
$$

and using (1.2) this conservation property can be rewritten as

$$
\frac{d}{d t} \int_{\Omega_{1}} u d x-\int_{\Gamma}\{\varepsilon \nabla u \cdot \mathbf{n}\} d s=\int_{\Omega_{1}} f d x
$$

For the discretization we obtain (using a suitable test function $v_{h}$ ):

$$
\frac{d}{d t} \int_{\Omega_{1}} u_{h} d x-\int_{\Gamma}\left\{\varepsilon \nabla u_{h} \cdot \mathbf{n}\right\} d s-\frac{\lambda \varepsilon}{h} \int_{\Gamma}\left[\beta u_{h}\right] d s=\int_{\Omega_{1}} f d x .
$$

Hence there is a discrepancy between $\int_{\Omega_{1}} u d x$ and $\int_{\Omega_{1}} u_{h} d x$ that is controlled by $\left\|\left\{\varepsilon \nabla e_{h} \cdot \mathbf{n}\right\}\right\|_{L^{2}(\Gamma)}$ and $\frac{\lambda \varepsilon}{h}\left\|\left[\beta e_{h}\right]\right\|_{L^{2}(\Gamma)}, e_{h}:=u-u_{h}$. From the error analysis it folows that both terms tend to zero for $h \downarrow 0$, i.e., we have a consistency property.

REMARK 6. In view of applications the case of a non-stationary interface $\Gamma(t)$ is much more interesting than that of a stationary one. We comment on a generalization of the method in (2.16) to the former case. For an evolving interface $\Gamma(t)$, instead of the weak formulation in (2.4), one has to consider a space-time variational formulation to obtain a well-posed problem, cf. [9]. A discretization of the time derivative by means of finite difference approximations (as done here for a stationary interface) does no longer lead to a consistent discretization if the interface $\Gamma(t)$ is moving in time. A discretization based on a space-time formulation using a suitable space-time extended finite element space should be used. For a convection dominated problem this can be combined with a space-time streamline diffusion stabilization. The development and analysis of such a space-time SD-Nitsche-XFEM method is a topic of current research.

Acknowledgement. The authors gratefully acknowledge funding by the German Science Foundation (DFG) within the Priority Program (SPP) 1506 "Transport Processes at Fluidic Interfaces".

REFERENCES 
[1] R. Becker, E. Burman, And P. Hansbo, A Nitsche extended finite element method for incompressible elasticity with discontinuous modulus of elasticity, Comput. Methods Appl. Mech. Engrg., 198 (2009), pp. 3352-3360.

[2] T. Belytschko, N. Moes, S. Usui, and C. Parimi, Arbitrary discontinuities in finite elements, Int. J. Num. Meth. Eng., 50 (2001), pp. 993-1013.

[3] D. Bothe, M. Koebe, K. Wielage, J. Prüss, and H.-J. Warnecke, Direct numerical simulation of mass transfer between rising gas bubbles and water, in Bubbly Flows: Analysis, Modelling and Calculation, M. Sommerfeld, ed., Heat and Mass Transfer, Springer, 2004.

[4] D. Bothe, M. Koebe, K. Wielage, and H.-J. Warnecke, VOF-simulations of mass transfer from single bubbles and bubble chains rising in aqueous solutions, in Proceedings 2003 ASME joint U.S.-European Fluids Eng. Conf., Honolulu, 2003, ASME. FEDSM2003-45155.

[5] A. Chernov and P. Hansbo, An hp-Nitsche's method for interface problems with nonconforming unstructured finite element meshes, Lect. Notes Comput. Sci. Eng., 76 (2011), pp. 153-161.

[6] J. Chessa and T. Belytschko, An extended finite element method for two-phase fluids, ASME Journal of Applied Mechanics, 70 (2003), pp. 10-17.

[7] H. Elman, D. Silvester, and A. Wathen, Finite Elements and Fast Iterative Solvers, Oxford University Press, Oxford, 2005.

[8] S. Gross, V. Reichelt, And A. Reusken, A finite element based level set method for two-phase incompressible flows, Comp. Visual. Sci., 9 (2006), pp. 239-257.

[9] S. Gross And A. Reusken, Numerical Methods for Two-phase Incompressible Flows, Springer, Berlin, 2011.

[10] A. Hansbo and P. Hansbo, An unfitted finite element method, based on nitsche's method, for elliptic interface problems, Comput. Methods Appl. Mech. Engrg., 191 (2002), pp. 55375552 .

[11] _ - A finite element method for the simulation of strong and weak discontinuities in solid mechanics, Comput. Methods Appl. Mech. Engrg., 193 (2004), pp. 3523-3540.

[12] A. Hansbo, P. Hansbo, and M. Larson, A finite element method on composite grids based on Nitsche's method, Math. Model. Numer. Anal., 37 (2003), pp. 495-514.

[13] P. Hansbo, C. Lovadina, I. Perugia, and G. Sangalli, A Lagrange multiplier method for the finite element solution of elliptic interface problems using non-matching meshes., Numer. Math., 100 (2005), pp. 91-115.

[14] M. Ishi, Thermo-Fluid Dynamic Theory of Two-Phase Flow, Eyrolles, Paris, 1975.

[15] M. Muradoglu and G. Tryggvason, A front-tracking method for computation of interfacial flows with soluble surfactant, J. Comput. Phys., 227 (2008), pp. 2238-2262.

[16] A. Reusken, Analysis of an extended pressure finite element space for two-phase incompressible flows, Comp. Visual. Sci., 11 (2008), pp. 293-305.

[17] A. Reusken and T. NGuYen, Nitsche's method for a transport problem in two-phase incompressible flows, J. Fourier Anal. Appl., 15 (2009), pp. 663-683.

[18] H.-G. Roos, M. Stynes, and L. Tobiska, Numerical Methods for Singularly Perturbed Differential Equations - Convection-Diffusion and Flow Problems, vol. 24 of Springer Series in Computational Mathematics, Springer-Verlag, Berlin, second ed., 2008.

[19] S. Sadhal, P. Ayyaswamy, and J. Chung, Transport Phenomena with Droplets and Bubbles, Springer, New York, 1997.

[20] J. Slattery, Advanced Transport Phenomena, Cambridge Universtiy Press, Cambridge, 1999.

[21] A.-K. Tornberg And B. Engquist, A finite element based level-set method for multiphase flow applications, Comp. Vis. Sci., 3 (2000), pp. 93-101.

[22] F. Verhulst, Methods and Applications of Singularly Perturbations - Boundary Layers and Multiple Timescale Dynamics, vol. 50 of Texts in Applied Mathematics, Springer-Verlag, Berlin, first ed., 2000. 